\title{
On an intuitionistic logic for pragmatics
}

\author{
Gianluigi Bellin*, Massimiliano Carrara ${ }^{\circ}$ and Daniele Chiffi ${ }^{\S}$ \\ (*) Dipartimento di Informatica, Università di Verona, \\ Strada Le Grazie, 37134 Verona, Italy \\ gianluigi.bellin@univr.it and \\ (o) Department of Philosophy, University of Padua and \\ (§) DMM, University of Padua
}

Summary. We reconsider the pragmatic interpretation of intuitionistic logic [21] regarded as a logic of assertions and their justifications and its relations with classical logic. We recall an extension of this approach to a logic dealing with assertions and obligations, related by a notion of causal implication [14, 45]. We focus on the extension to co-intuitionistic logic, seen as a logic of hypotheses [8, 9, 13] and on polarized bi-intuitionistic logic as a logic of assertions and conjectures: looking at the S4 modal translation, we give a definition of a system AHL of bi-intuitionistic logic that correctly represents the duality between intuitionistic and co-intuitionistic logic, correcting a mistake in previous work $[7,10]$. A computational interpretation of cointuitionism as a distributed calculus of coroutines is then used to give an operational interpretation of subtraction. Work on linear co-intuitionism is then recalled, a linear calculus of co-intuitionistic coroutines is defined and a probabilistic interpretation of linear co-intuitionism is given as in [9]. Also we remark that by extending the language of intuitionistic logic we can express the notion of expectation, an assertion that in all situations the truth of $p$ is possible and that in a logic of expectations the law of double negation holds. Similarly, extending co-intuitionistic logic, we can express the notion of conjecture that $p$, defined as a hypothesis that in some situation the truth of $p$ is epistemically necessary.

to Carlo Dalla Pozza (1942-2014)

\section{Preface: intuitionistic pragmatics and its extensions.}

Conceptually, this paper is about a logico-philosophical framework called Logic for Pragmatics, initiated by Carlo Dalla Pozza and Claudio Garola [21], to represent intuitionistic Logic (IL) as a logic of assertions and justifiability and also to specify the relations of intuitionistic logic with Classical Logic (CL) as a logic of propositions and truth ${ }^{1}$ Asserting, making hypotheses, establishing

\footnotetext{
${ }^{1}$ Carlo Dalla Pozza has been a significant and stimulating presence in Italian philosophical logic and also a good friend. He had an unusual breadth of intellectual
} 
obligations, asking questions are illocutionary acts [6]; their study is regarded as belonging to pragmatics, thus such a representation of intuitionism may be seen as the beginning of a logical study of pragmatic phenomena. In the Dalla Pozza and Garola's framework, a declarative sentence results from an application of the illocutionary force of assertion to a proposition, classically understood. Such a sentence is formally represented as $\vdash p$.

The language $\mathcal{L}^{P}$ of the logic for pragmatics is based on classical propositional logic, built from atomic propositions $p$, the propositional constants true $T$ and false $\perp$ with classical connectives $(\wedge, \vee, \rightarrow, \neg)$, but it has also pragmatics connectives, building sentences from assertions, rather than propositions; such connectives are interpreted intuitionistically. Namely, intuitionistic assertive formulas $A$ are built from elementary formulas $\vdash p$, the type of assertions of $p$, an assertion $\curlywedge$ which is always justified, with intuitionistic conjunction $(\cap)$, implication $(\supset)$, disjunction $(\cup)$ and negation $(\sim A)$. A semantics for such a language can be given through the familiar translation of IL into classical S4, epistemically understood, due to Gödel [28], McKinsey and Tarski [33]:

$$
\begin{array}{cc}
(\vdash p)^{M}=\square p, & (A \supset B)^{M}=\square\left(A^{M} \rightarrow B^{M}\right), \\
(\curlyvee)^{M}=\top, & (\sim A)^{M}=\square \neg A^{M}, \\
(A \cap B)^{M}=A^{M} \wedge B^{M}, & (A \cup B)^{M}=A^{M} \vee B^{M} .
\end{array}
$$

This translation allows us to obtain Kripke's possible world semantics for IL from the possible world semantics for classical S4 [31]. However our basic understanding of intuitionistic logic is based not on Kripke's semantics, but on the Brouwer-Heyting-Kolmogorov interpretation (BHK) which takes the informal notion of proof and of mathematical method as fundamental: an elementary formula $p$ is interpreted through its informal proofs, a proof of an implication $A \supset B$ is a method transforming a proof of $A$ into a proof of $B$, there is no proof of the absurdity, and so on. This is also the interpretation of intuitionistic pragmatic connectives in Dalla Pozza and Garola's logic.

We reconsider an extension of the logic for pragmatics to co-intuitionistic logic $[7,8,9,13]$, thought of as a logic of hypotheses. Co-intuitionistic hypothetical formulas $C$ are built from $\mathcal{H} p$, the type of hypothesis that $p$ may be true and a hypothesis $\curlywedge$ which is never justified with disjunction $(\curlyvee)$, subtraction $(\backslash)$ and co-intuitionistic negation (supplement) $\frown C$. These connectives are dual of the intuitionistic ones. The $\mathbf{S} 4$ interpretation of co-intuitionistic logic in [7] is as follows:

$$
\begin{array}{cc}
(\mathcal{H} p)^{M}=\diamond p, & (C \backslash D)^{M}=\diamond\left(C^{M} \wedge \neg D^{M}\right), \\
(\curlywedge)^{M}=\perp, & (\frown C)^{M}=\diamond \neg C^{M}, \\
(C \curlyvee D)^{M}=C^{M} \vee D^{M}, & (C \curlywedge D)^{M}=C^{M} \wedge D^{M} .
\end{array}
$$

Notice that the dual of $\vdash p$ is $\mathcal{H} \neg p$, not $\mathcal{H} p$ : hence definition in (1) is correct for a treatment of co-intuitionism but is not suitable to represent

interests, including philosophy of science and of laws, and was a generous person, as a man and as researcher. We remember here his intelligence and generosity. 
the duality between intuitionistic and co-intuitionistic logic. For this purpose let $\left\{p_{0}, p_{0}^{*}, p_{1}, p_{1}^{*}, \ldots\right\}$ be a sequence of propositional atoms. We set $\vdash p_{i}$ in intuitionistic language, but $\mathcal{H} p_{i}^{*}$ in the co-intuitionistic one and in the modal translation we let $\left(\mathcal{H} p_{i}^{*}\right)^{\perp}=\diamond \neg p_{i}$. Then the $\mathbf{S} 4$ interpretation of co-intuitionistic logic becomes dual to that of intuitionistic logic:

$$
\begin{aligned}
& \left(\mathcal{H} p^{*}\right)^{M}=\diamond \neg p, \quad(C \backslash D)^{M}=\diamond\left(C^{M} \wedge \neg D^{M}\right), \\
& (\curlywedge)^{M}=\perp, \quad(\frown C)^{M}=\diamond \neg C^{M} \text {, } \\
& (C \curlyvee D)^{M}=C^{M} \vee D^{M}, \quad(C \curlywedge D)^{M}=C^{M} \wedge D^{M} .
\end{aligned}
$$

Here however the co-intuitionistic side is no longer a logic of hypotheses, but of negative hypotheses about a proposition $p$, i.e., objections to the assertability of $p$. Here we face the problem of extending the BHK interpretation to the logic of hypotheses.

We also look at the pragmatic interpretation of bi-intuitionistic logic as a logic of assertions and hypotheses (AHL) proposed in $[8,10]$ with modal translation in $\mathbf{S} \mathbf{4}$ and at the sequent calculus $\mathbf{A H}-\mathbf{G} \mathbf{1}$ which extends intuitionistic and co-intuitionistic logic with two negations, both denoted by ()$^{\perp}$, relating the two sides. Using the definition in (2) instead of that in (1), our "polarized" bi-intuitionistic logic is a logic of assertions and objections and does represent the duality between intuitionistic and co-intuitionistic logic.

Expectations are defined in [8] as elementary expressions $\mathcal{E} p$ that have modal translation $(\mathcal{E} p)^{M}=\square \diamond p$. Let $\mathcal{L}^{E}$ be the language generated by elementary expressions $\mathcal{E} p$ with intuitionistic conjunction, implication and negation only. It is easy to show that the set of $\mathcal{L}^{E}$ formulas that are valid with respect to the modal translation is closed for the classical double negation law.

$$
\sim E \Rightarrow E .
$$

(Exactly the same holds for expectations of objections $\mathcal{E} p^{*}$.) This is a pragmatic interpretation of the $\square \diamond$ modal translation of propositional classical logic [52], but without disjunction, which therefore retains the intuitionistic interpretations of the connectives and satisfies the double negation rule.

Opposite of expectations are conjectures that have modal translation $(\mathcal{C} p)^{M}=$ $\diamond \square p$.

There is a tradition of polarization of classical logic and linear logic starting from J-Y. Girard fundamental paper [25] and continued in many ways particularly in the work by O. Laurent in relation to game semantics whose significance for the study of bi-intuitionism should be explicitly discussed. We have to leave this for future work. 


\section{Part I. Intuitionistic pragmatics: a philosophical overview.}

Carlo Dalla Pozza and Claudio Garola's pragmatic interpretation [21] presents intuitionistic logic as a logic of assertions, following suggestions by Michael Dummett; however, intuitionistic pragmatics is given in a two-layers formal system where classical semantics is also represented; broadly speaking, their goal is to show how intuitionism can be reconciled with classical logic. The grammar of the formal language $\mathcal{L}^{P}$ of the logic for pragmatics is a follows:

$$
\begin{aligned}
\alpha & :=p|\top| \perp|\neg \alpha| \alpha_{1} \rightarrow \alpha_{2}\left|\alpha_{1} \wedge \alpha_{2}\right| \alpha_{1} \vee \alpha_{2} \\
A, B & :=\quad r \alpha|\curlyvee| \sim A|A \supset B| A \cap B \mid A \cup B
\end{aligned}
$$

Negation can be defined as $\sim A=_{d f} A \supset \mathbf{u}$ where $\mathbf{u}$ is a assertions that is always unjustified.

The elementary formulas of Dalla Pozza and Garola's formal language $\mathcal{L}^{P}$ have the form $\vdash \alpha$ where Frege's symbol " $\vdash$ " represents an (impersonal) illocutionary force of assertion and $\alpha$ is a propositional formula interpreted in classical truth-functional semantics. Thus in accordance with Frege propositions are classically true or false; illocutionary acts of assertions can only be justified or unjustified. However, unlike in Frege, there are pragmatic connectives that build up formulas from elementary assertions. Such pragmatic expressions are interpreted according to the Brouwer-Heyting-Kolmogorov (BHK) interpretation of intuitionistic connectives. In this framework the BHK interpretation is as follows:

- $\vdash \alpha$, an assertion that $p$, is justified by a proof (or conclusive evidence) of the truth of $\alpha$;

- $A \supset B$ is justified by a method transforming a justification of the sentence $A$ into a justification of the sentence $B$;

- $A \cap B$ is justified by a pair $\left\langle e_{1}, e_{2}\right\rangle$ of justifications, $e_{1}$ of $A$ and $e_{2}$ of $B$;

- $A_{0} \cup A_{1}$ is justified by a pair $\langle e, i\rangle$ where $i=0,1$ and $e$ is a justification of $A_{i}$

- $\sim A$ is justified by a method transforming a proof of $A$ into an absurdity.

Every pragmatic expression of Dalla Pozza and Garola's formal language $\mathcal{L}^{P}$ has justification conditions in accordance with the BHK interpretation. An expression meeting such conditions is justified; otherwise it is unjustified. In any elementary expression $\vdash \alpha$ the radical part $\alpha$ is a proposition in the sense of Frege and has a truth value true or false in the sense of classical logic; moreover, such a semantic value contributes to determine whether or not the justification conditions for $\vdash \alpha$ are met.

Pragmatic expressions $A$ have a classical semantic value through Gödel, McKinsey and Tarski's translation of intuitionistic logic into the classical modal logic S4: 


$$
\begin{array}{cc}
(\vdash \alpha)^{M}=\square \alpha, & (A \supset B)^{M}=\square\left(A^{M} \rightarrow B^{M}\right), \\
(\curlyvee)^{M}=\top, & (\sim A)^{M}=\square \neg A^{M}, \\
(A \cap B)^{M}=A^{M} \wedge B^{M}, & (A \cup B)^{M}=A^{M} \vee B^{M} .
\end{array}
$$

To obtain a representation of intuitionistic logic in language $\mathcal{L}^{P}$ Dalla Pozza and Garola consider the set of formulas $A$ whose elementary expressions have the form $\vdash p$ with $p$ is atomic. In this way the justification value of $A$ depends only on the BHK interpretation and not on the classical structure of the radical parts of elementary expressions.

\subsection{Classical constructivism or intuitionistic pragmatics?}

However, the informal Brouwer-Heyting-Kolmogorov's interpretation of connectives can also be developed in a classical constructive interpretation. This would be true of interpretations taking the $\mathbf{S} \mathbf{4}$ translation of intuitionistic logic as defining its meaning. We may call this kind of interpretations reductive.

Reductive interpretations are among the first models of intuitionism. As pointed out by Artemov [3], section 2, "Kolmogorov in 1932 (and then Gödel in 1933) intended to interpret Int on the basis of the usual mathematical notion of proof (problem solution), and thus to provide a definition of Int within classical mathematics independent of intuitionistic assumptions." Artemov follows this approach in the paper Explicit Provability and Constructive Semantics [3]. There he defines the system LP, Logic of proofs, whose expressions $t: A$ realise the necessity operator $\square A$ of $\mathbf{S} 4$. Here $t$ is a "proof polynomial", built from variables and constants with the operation of application $(t \cdot s)$, sum $(s+t)$ and proof checker ! $t$ and the formula $A$ may be a dependent type expression in the case of the axiom $t: F \rightarrow ! t:(t: F)$ that realises the $\mathbf{S} \mathbf{4} \square A \rightarrow \square \square A$. Then Artemov gives a provability interpretation of $\mathbf{L P}$ in Peano Arithmetic. (This result is the analogue or Solovay's interpretation in PA of the Gödel-Löb GL modal logic of arithmetic provability.) The point here is that an intuitionistic proof $d$ of $A$ is first translated into a proof polynomial $t: A^{\prime}$ and then interpreted as an arithmetic expression of classical PA. Thus Artemov can claim that this work completes Gödel's representation of intuitionistic formulas in $\mathbf{S} 4$ by giving also an interpretation of intuitionistic proofs in a classical provability interpretation of $\mathbf{S} \mathbf{4}$. Intuitionism is thus reduced to classical constructivism.

We quoted an example of reductive interpretation in the Logic of Prooofs, but S. Artemov and R. Iemhoff have also given interpretations of intuitionistic logic in Heyting arithmetic [4]. Justification Logics is a large research area and we have no pretence to survey it here. Comparison with the approach of the Logic for pragmatics is left for future work.

The intentions of Dalla Pozza and Garola are in fact reductive and thus we can say that Artemov's classical LP provides a very sophisticated development of such a project. However, if the logic for pragmatics has also to serve 
the purpose of representing intuitionism and its difference from classical logic, then we must make sure that the logical representation of intuitionistic logic within it does satisfy the requirements of an intuitionistic philosophy. Unfortunately this is not clear: the meta-theory of Dalla Pozza and Garola's logic for pragmatics makes essential use of classical logic.

But can the logic for pragmatics represent intuitionism without reducing it to classical constructivism? A concern is mathematical: one would like to think of a logic for pragmatics as open to modern results of proof theory, such as the Curry-Howard correspondence, type theory and categorical logic: a good part of these subjects belongs to intuitionistic mathematics.

It must be said that Dalla Pozza's attitude has never been dogmatic with respect to the application of his framework. The main goal is to present different logics with an intended interpretation with respect to actual linguistic usage and thus clarify their relations, as it is in the case of intuitionistic pragmatics and classical semantics. But most of mathematics is not logic and it stands on its own: only in some cases we can find intended interpretations of formal systems or show how a formal systems, seen as theoretical construction, can be related to linguistic practice (see the treatment of relevant and linear logic in [11]) .

Another concern is philosophical, in particular two of Dalla Pozza's philosophical theses are typical of the use of classical logic in the meta-theory.

(1) Propositions are only those of classical logic.

(2) Illocutionary forces apply only to classical propositions.

By (2), a justification of $\vdash p$ is conclusive evidence of the truth of $p$ in the classical interpretation of truth. Thus the entire construction seems to rely on an understanding of $p$ as a classical proposition.

One may say that an intuitionistically adequate representation of intuitionism require the use of an intuitionistic meta-theory. ${ }^{2}$. We may also allow only propositions $p$ that are intuitionistically acceptable in elementary formulas $\vdash p$ : in an intuitionistic approach, their justification value depends on the verification conditions for $p$, which are given by the meaning of $p$. If $p$ is intuitionistically unacceptable then $\vdash p$ is a void assertion. How much is changed in Dalla Pozza and Garola's framework [21] if one takes this route?

In accordance with the Curry-Howard correspondence, we can say that sentences of the logic for pragmatics can always seen as types of their justification values. In any type system (e.g., the simply typed lambda calculus with products) terms are representation of intuitionistic proofs and types are the intuitionistic proposition which is proved, thus a natural form of justification terms for the logic for pragmatics is that of a typed $\lambda$-term of the form $t: A$

\footnotetext{
${ }^{2}$ We do not adopt this method: in this paper we use classical modal logic extensively in our discussion. Use of constructive $\mathbf{S} 4$ would otherwise be required in an intuitionistic meta-theory.
} 
where $t$ represents a proof of $A$. Since terms encode proofs, in the representation of proofs by $\lambda$-terms "nothing is added or reduced". But in Martin-Löf type theory to say that a proposition, regarded as a type, is true amounts to say that it is inhabited by a term representing its proof, i.e., truth coincides with provability. Clearly any true proposition can be asserted. Hence all the distinctions established by the logic for pragmatics are trivialized.

Thus it seems that even in the most abstract form an intuitionistic logic for pragmatics becomes useful when a distinction can be made between the justification conditions of a particular class of elementary expressions and for the non-elementary ones. A distinctive contribution of the logic for pragmatics, both in the "reductive" and in the "intuitionistic" version, has been to highlight the role of the illocutionary force occurring in elementary expressions of natural language in determining the logical properties of common sense reasoning with those expressions: indeed what counts as a justification of a sentence essentially depends on whether its elementary components are assertions, hypotheses, questions, answers, commands and so on. Different illocutionary acts have different logical properties.

This is the case of work by Kurt Ranalter, developing ideas by Bellin and Dalla Pozza [11] on a logic of assertions, obligations and causal implication, represented as relevant implication, in the framework of the intuitionistic logic for pragmatics. Such a logic provides innovative tools for representing deontic reasoning, modelled first by Kripke-style semantics [14] and then developed in Ranalter's thesis [44] into a categorical model [45].

This is evident also considering assertions and hypotheses, as we shall see. Moreover, different forms of negations, such as the intuitionistic negation (denial) $(\sim A)$ of a sentence $A$ or the co-intuitionistic supplement, to be interpreted as doubt $(\curvearrowleft C$ ) that $C$ may be false, may be related to a basic distinction between assertive and hypothetical reasoning. Thus the law of excluded middle $C \curlyvee \frown C$ for hypothetical expressions has different justification values than its corresponding form $A \cup \sim A$, where is assertive.

However, in natural language assertions are mostly about empirical facts justified by empirical evidence which comes with different degrees of strength. It seems that what's needed to justify an assertion is only conclusive evidence; but what counts as 'conclusive evidence' may depend on the subject matter. In the framework of the logic for pragmatics, one would like assertions of elementary formulas to satisfy the Factivity Axiom: if $p$ is assertible, then $p$ is true. But very often common sense reasoning is not grounded in conclusive evidence and factivity does not hold. This is the domain of a logic of hypotheses, which may be justified already by non-conclusive evidence.

In the representation of intuitionistic logic elementary expressions $\vdash \alpha$ must have an atomic radical part $\alpha$. Does this restriction suffice to guarantee that intuitionistic logic is adequately represented in this logic for pragmatics? Actually, Dalla Pozza considers the case of propositions $p$ that have a semantic 
value true or false, but no evidence at all may be available of this fact. They are called intrinsically undecidable or pragmatically undecidable sentences.

Example 1. A statement by Jules-Henri Poincaré ([39], Book 2, chapter 1) is often quoted, that all bodies in the universe expand continuously and uniformly - including the magnitudes we take as standards of measurement. There can be no conclusive justification for asserting this statement nor for asserting its negation. (We do not take into account here the argument that observation of a non-uniform expansion would constitute an experimental test against the statement.)

From the viewpoint of an intuitionistic philosophy, as expressed by Michael Dummett [23], intrinsically undecidable statements have no definite truth value; in fact, if we cannot specify conditions in which we would be able to obtain evidence of their truth, they must be considered meaningless. However Dummett does not propose to eliminate the property of statements of being true in favour of being justifiably assertible: the notion of truth carries a connotation of objectivity that must be retained. Thus Dummett advocates a notion of intuitionistic truth which may be applied only to assertive statements having definite justification conditions.

\section{Intermezzo: Assertions, Obligations and Causal Inference}

An application of the intuitionistic logic for pragmatics to common sense reasoning and deontic logic comes from the paper [11] and its proof theoretic treatment of Assertions, Obligations and Causal Implication. This work has been developed by Kurt Ranalter's dissertation and papers [14, 44, 45].

The logic, called $\mathbf{I L P} \mathbf{P}_{A O C}$, deals with the properties of impersonal acts of assertion and acts of obligation, and relates them through a notion of causal implication, regarded as a fragment of relevant logic. Kurt Ranalter started by giving a Kripke semantics for ILP $_{A O C}$ proves a completeness theorem, decidability and finite model property [14]. Then the system was refined it to show that it is sound and complete with respect to a class of algebraic, Kripke and categorical models $[44,45]$. This work has an interest for linguistics and the representation of common sense reasoning. It gives a rigorous framework for deontic reasoning with respect to causal inference and assertive reasoning. It provides an example of an abstract categorical representation that unifies a complex structure, involving different forms of deduction represented by different consequence relations. We cannot discuss here the semantics and sketch here only the syntax to illustrate possible linguistic applications.

We have an intuitionistic system of logic for pragmatics, formalized with two consequence relations, intuitionistic and intuitionistic relevant, let's call it ILP $_{A O C}$. The purpose of this calculus is to formalize inferences of the following forms: 
Suppose the gun is loaded and pulling the trigger causes killing a polar bear.

(1.1) Hence, if one ought to pull the trigger then they ought to kill a bear. Similarly, we can infer

(1.2) Hence, if it is forbidden to kill a bear then it is forbidden to pull the trigger.

(3.1) Hence, if it is permissible to pull the trigger then it is permissible to kill a bear.

We focus on (1.1). Here we have the description of a causal chain of events by a conditional of the form if the gun is loaded $(L)$ and the trigger is pulled $(T)$, then a bear is killed $(K)$, formally represented by the formula $\zeta$ :

$$
\zeta: \quad \vdash L \supset_{c}\left(\vdash T \supset_{c} \vdash K\right) .
$$

The inference (1.1) is then formalized as follows:

$$
\frac{\vdash L, \vdash T, \vdash L \supset_{c}\left(\vdash T \supset_{c} \vdash K\right) \Rightarrow \vdash K}{\vdash L, \mathcal{O} T, \vdash L \supset_{c}\left(\vdash T \supset_{c} \vdash K\right) \Rightarrow \mathcal{O} K}
$$

Causal implication is thought to hold between events $e_{1}, \ldots, e_{n}$ and an event $e$ whenever $e$ follows from $e_{1}, \ldots, e_{n}$ in accordance with a natural law. Here events $e_{1}, e_{2}$ and $e$ are described by assertions $\vdash L$ and $\vdash T$ and $\vdash K$ and we have a method, namely a causal law, allowing us to transform a justification of $\vdash L$ and $\vdash T$ into a justification of $\vdash K$.

The notion of causality considered here is further specified as follows. First, only an event-describing assertion can be the antecedent of a causal implication. Second, neither the antecedent nor the consequent of a causal implication can be the expression of an obligation. For instance, we can write $\vdash L \supset(\mathcal{O} T \supset \mathcal{O} K)$, but using causal rather than intuitionistic implication would be an extension of the notion of causality which is regarded as ungrammatical here.

Third, the main property of causal implication is that it is relevant, i.e., nonmonotonic. Let $e_{1}, e_{2}$ and $e_{3}$ be described by $\vdash p, \vdash q$ and $\vdash r$ and suppose $e_{2}$ is a causal precondition of $e_{1}$ but $e_{3}$ is not. Then it must be incorrect to infer $r \supset_{c}\left(\vdash q \supset_{c} \vdash p\right)$ from $\vdash q \supset_{c} \vdash p$. This fact is best described as a property of the consequence relation " $\Rightarrow$ " in which we express causal inferences, namely, by saying that a causal consequence relation is non-monotonic. It follows that in a Gentzen system for causal implication we cannot admit the rule of weakening, that would allow us to introduce $\vdash r$ in the antecedent. On the other hand, the rule of contraction is permissible: an event $e$ can have multiple consequences and there is no reason to restrict the number of uses of its description $\vdash p$ in an inferential process.

The above fragment of causal and deontic reasoning is formalized by the following grammar and sequent calculus. Let $p_{1}, p_{2}, \ldots$ be an infinite sequence of propositional letters. 


$$
\begin{array}{ll}
\eta:=\vdash p_{i} & \text { (assertive elementary expressions) } \\
\xi:=\eta & \mid \eta \supset_{c} \xi \text { (causal expressions) } \\
\gamma:=\mathcal{O} p \mid \xi & \text { (causal deontic expressions) }
\end{array}
$$

We use $\eta_{p}$ as a metavariable for the assertive elementary expression $\vdash p$ and $\eta_{p}^{o}$ for the deontic expession $\mathcal{O}$ p. $\Xi$ and $\Gamma$ denote sequences of causal and causal deontic expressions, respectively.

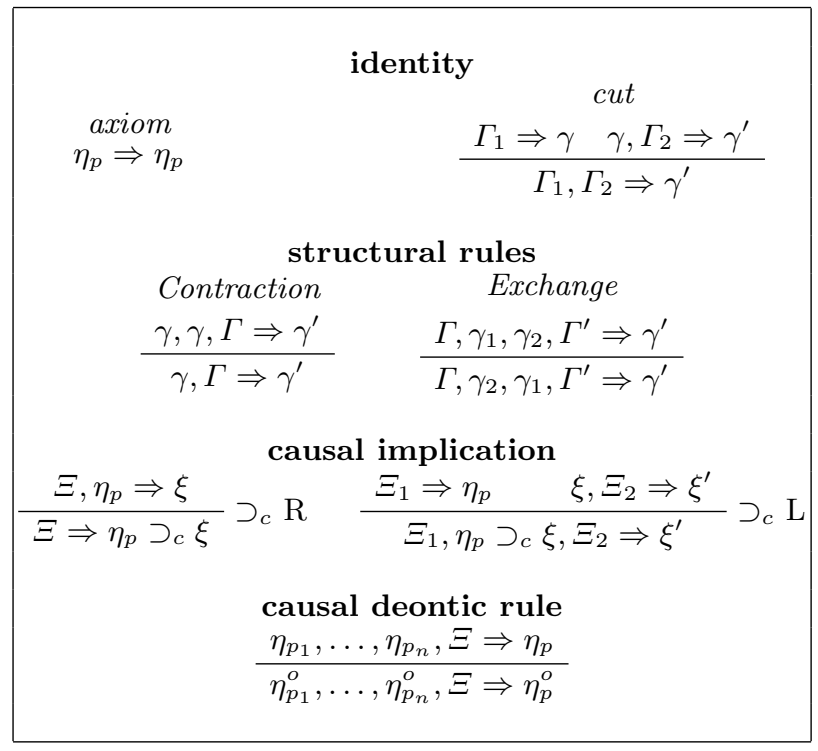

It is possible to combine causal-deontic and intuitionistic reasoning by extending the grammar and the sequent calculus as follows. We need sequents with two areas in the antecedent of the form

$$
\underbrace{\Gamma}_{\text {relevant }} ; \underbrace{\Delta}_{\text {intuitionistic }} \Rightarrow \delta
$$

where formulas in $\Gamma$ are causal deontic and in $\Delta$ intuitionistic. The rule of weakening is allowed only in the intuitionistic area, contraction and exchange in both. In addition, there is a rule of dereliction:

$$
\frac{\Gamma, \gamma ; \Delta \Rightarrow \delta}{\Gamma ; \gamma, \Delta \Rightarrow \delta}
$$

\section{$4 \mathrm{Bi}$-Intuitionistic logic}

Dalla Pozza's approach has been extended to co-intuitionistic logic, interpreted as a logic of hypothetical reasoning, which is thought of as symmetric to assertive reasoning. Co-intuitionism has been studied in the $\mathbf{S} \mathbf{4}$ translation, in proof theory with term assignments $[15,8,13]$ and on a categorical model of 
co-intuitionism [9]. An interpretation of bi-intuitionistic logic as a logic AHL of assertions and hypotheses has also be proposed [7, 8, 10], where assertive intuitionistic logic and hypothetical co-intuitionistic logic are combined with two dual negations, both denoted by ()$^{\perp}$, relating the two sides. A sequent calculus, called AH-G1 here, gives an axiomatization of AHL (see the Appendix, Section 9). However, there is an obvious mistake in the definition of AHL in the prevous papers that we correct here.

For another sequent calculus axiomatizing AHL, called $\mathbf{I L P}_{A C}$ (Intuitionistic Logic for Pragmatics of Assertions and Conjectures), Biasi and Aschieri [15] gave a term calculus in the style of Herbelin and Curien $\lambda \bar{\lambda}$ calculus. They proved strong normalization of this term calculus by reducing it to the simply typed $\lambda$ calculus with pairing and projections. Their term calculus represents justification conditions for intuitionistic assertions but refutation conditions for co-intuitionistic hypotheses. As a consequence, there is an isomorphism between a proof of an assertive formula $A$ and a refutation of the

The interest in the subject has come from the first author. We must say that although Carlo Dalla Pozza has been informed of these researches and their conceptual content, he has always manifested his view that a theory of hypotheses and conjectures should be developed in a probabilistic framework and in a Bayesian theory of learning rather than by developing a pragmatic theory of co-intuitionistic logic with the tools used for the pragmatic interpretation of intuitionism. On this topic the book [22] is about to be published posthumously.

\subsection{Bi-Heyting algebras and categorical models of bi-intuitionism.}

The mathematical theory of co-intuitionistic logic, initiated by Cecylia Rauszer $[46,47]$ and promoted among others by William Lawvere [32], begins by dualizing the language and the known algebraic semantics of intuitionistic logic.

Definition 1. A Heyting algebra is a bounded lattice $\mathcal{A}=(A, \vee, \wedge, 0,1)$ (namely, with join and meet operations, the least and greatest element), and with a binary operation, Heyting implication $(\rightarrow)$, which is defined as the right adjoint to meet. A co-Heyting algebra is a lattice $\mathcal{C}$ such that its opposite $\mathcal{C}^{o p}$ (reversing the order) is a Heyting algebra. $\mathcal{C}$ has structure $(C, \vee, \wedge, 1,0)$ with an operation of subtraction $(\backslash)$ defined as the left adjoint of join. Thus we have the rules

$$
\begin{array}{lc}
\text { Heyting algebra } & \text { co-Heyting algebra } \\
c \wedge b \leq a & a \leq b \vee c \\
\hline \hline c \leq b \rightarrow a & \frac{a \leq b \leq c}{a \backslash b}
\end{array}
$$

A bi-Heyting algebra is a lattice that has both the structure of Heyting and of a co-Heyting algebra. 
Definition 2. (Rauszer's Kripke semantics) Kripke models for bi-intuitionistic logic have the form $\mathcal{M}=(W, \leq, \Vdash)$ where the accessibility relation $\leq$ is reflexive and transitive, and the forcing relation "|-" satisfies the usual conditions for $\vee, \wedge, 0$ and 1 and moreover

$$
\begin{aligned}
& w \Vdash A \rightarrow B \text { iff } \forall w^{\prime} \geq w \cdot w^{\prime} \Vdash A \text { implies } w^{\prime} \Vdash B \text {; } \\
& w \Vdash A \backslash B \text { iff } \exists w^{\prime} \leq w \cdot w^{\prime} \Vdash A \text { and } w^{\prime} \| B \text {. }
\end{aligned}
$$

Such conditions guarantee the monotonicity property for all bi-intuitionistic formulas. Informally, they could be explained by saying that implication has to hold in all possible worlds "in the future of our knowledge" and subtraction in some world "in the past of our knowledge". In fact Rauszer's Kripke semantics for bi-intuitionistic logic is associated with a modal translation into (what is called today) tensed $\mathbf{S} 4$.

One would like to generalize these notions to a categorical semantics for biintuitionistic logic, but a result by Tristan Crolard [18] (Theorem 1.14) shows that any such categorical model would be trivial.

Theorem 1. If a Cartesian Closed Category $(C C C) \mathcal{C}$ is such that $\mathcal{C}^{o p}$ has also the structure of a CCC (namely, $\mathcal{C}$ has coproducts and co-exponents), then $\mathcal{C}$ is isomorphic to a partial order.

As a corollary, Crolard shows the following result.

Proposition 1. In the category Sets the coexponent $B_{A}$ of two sets $A$ and $B$ is defined if and only if $A=\emptyset$ or $B=\emptyset$.

The Theorem is a main motivation for "polarization", namely, for working with two categories, a CCC $\mathcal{A}$ and a co-CCC $\mathcal{B}$ modelling minimal intuitionistic logic and co-intuitionistic logic, respectively, related by two contravariant functors.

\section{Dualities}

In the proof theory of intuitionism and co-intuitionism the consequence relation are as follows:

$$
\begin{array}{cl}
\text { intuitionistic } & \text { co-intuitionistic } \\
A_{1}, \ldots, A_{n} \Rightarrow A & C \Rightarrow C_{1}, \ldots, C_{n}
\end{array}
$$

The following result applies to C.Rauszer's bi-intuitionistic logic and its modal translation.

Proposition 2. There is a duality ( )* between IL and co-IL with the following property. Let $\Gamma^{*}=C_{1}^{*}, \ldots, C_{n}^{*}$. In Rauszer's Kripke semantics $\Gamma \Rightarrow A$ is valid if and only if $A^{*} \Rightarrow \Gamma^{*}$ is valid. In particular $A$ is valid [contradictory] iff $A^{*}$ is contradictory [valid]. 
See Crolard [18], (Proposition 2.2. and theorem 2.14). Significant corollaries are the following:

1. Dual to assertive intuitionistic contradiction $A \cap \sim A$ is hypothetical excluded middle $A^{*} \curlyvee \frown A^{*}$, which is valid in co-intuitionistic logic;

2. Dual to assertive intuitionistic excluded middle $A \cup \sim A$ is hypothetical contradiction $A^{*} \curlywedge \frown A^{*}$, which is consistent in co-intuitionistic logic.

\subsection{Bi-intuitionistic pragmatic language.}

Here is our official definition of the extended pragmatic language $\mathcal{L}^{A H}$ of assertions and objections, amended from [10] by using the definition in (2) in place of that in (1) in Section 1.

Definition 3. (assertions, hypotheses) Let $\left\{p_{0}, p_{0}^{*}, p_{1}, p_{1}^{*}, \ldots\right\}$ be a sequence of propositional variables. For each pair $\left(p_{i}, p_{i}^{*}\right)$ we set $\vdash p_{i}$ and $\mathcal{H} p_{i}^{*}$.

- assertive:

$$
A, B:=\vdash p|\curlyvee| \mathbf{u}|A \supset B| A \cap B|A \cup B| C^{\perp}
$$

- hypothetical:

$$
C, D:=\mathcal{H} p^{*}|\curlywedge| \mathbf{j}|C \backslash D| C \curlyvee D|C \curlywedge D| A^{\perp}
$$

- defined negations:

$$
\sim A=_{d f} A \supset \mathbf{u} \quad \frown C={ }_{d f} \mathbf{j} \backslash C .
$$

Definition 4. (modal translation)

$$
\begin{array}{cc}
\text { assertive: } & \text { hypothetical: } \\
(\vdash p)^{M}=\square p & \left(\mathcal{H} p^{*}\right)^{M}=\diamond \neg p \\
(\curlyvee)^{M}=\top & (\curlywedge)^{M}=\perp \\
(\mathbf{u})^{M}=\perp & (\mathbf{j})=\top \\
(A \supset B)^{M}=\square\left(A^{M} \rightarrow B^{M}\right) & (C \backslash D)^{M}=\diamond\left(C^{M} \wedge \neg D^{M}\right) \\
(A \cap B)^{M}=A^{M} \wedge B^{M} & (C \curlyvee D)^{M}=C^{M} \vee D^{M} \\
(A \cup B)^{M}=A^{M} \vee B^{M} & (C \curlywedge D)^{M}=C^{M} \wedge D^{M} \\
\left(C^{\perp}\right)^{M}=\neg C^{M} & A^{\perp}=\neg\left(A^{M}\right) \\
\text { Defined negations: } & \\
(\sim A)^{M}=\square \neg A^{M} & \text { and }(\frown C)^{M}=\diamond \neg C^{M} .
\end{array}
$$

- Let $\mathcal{L}^{A}$ be the language generated by the above grammar for assertive formulas without the $(C)^{\perp}$ rule. $\mathcal{L}^{A}$ is the language of the intuitionistic logic for pragmatics, which we may identify with intuitionistic propositional logic Int.

- Similarly, let $\mathcal{L}^{H}$ be the language generated by the above grammar for hypothetical formulas without the $(A)^{\perp}$ rule. $\mathcal{L}^{H}$ is the language of the co-intuitionistic logic for pragmatics, which we may identify with cointuitionistic propositional logic co-Int.

- Let AHL be the logic defined by the language $\mathcal{L}^{A H}$ withe the semantics given by the modal translation into $\mathbf{S} \mathbf{4}$. 
A sequent calculus AH-G1 for the logic AHL is given in the Appendix, Section 9. It is sound and complete for the modal translation in $\mathbf{S} \mathbf{4}$.

In AHL the operations ()$^{\perp}$ in $(C)^{\perp}$ and $(A)^{\perp}$ are part of the language $\mathcal{L}^{A H}$. Their actions can be specified by the modal translation. Do they represent the meta-theoretic duality between intuitionism and co-intuitionism? The answer is yes. Consider the following equivalences:

$$
\begin{array}{ccc}
\text { (a) } & (\vdash p)^{\perp} \equiv \mathcal{H} p^{*} & \left(\mathcal{H} p^{*}\right)^{\perp} \equiv \vdash p \\
\text { (b) }(A \cap B)^{\perp} \equiv A^{\perp} \curlyvee B^{\perp} & (C \curlyvee D)^{\perp} \equiv C^{\perp} \cap D^{\perp} \\
\text { (c) }(A \supset B)^{\perp} \equiv B^{\perp} \backslash A^{\perp} & (C \backslash D)^{\perp} \equiv D^{\perp} \supset C^{\perp}
\end{array}
$$

Proposition 3. The equalities in definition 6 are satisfied by the modal translation in $\mathbf{S} 4$.

By Proposition 3 the dual negations internal to the language $\mathcal{L}^{A H}$ and metatheoretic duality agree. Thus we can use it to eliminate dual negations when possible.

Remark 1. Notice that if we use the definition (1) in Section 1 then the internal dual negations no longer correspond to the meta-theoretic duality between intuitionistic and co-intuitionistic logic under the $\mathbf{S} \mathbf{4}$ translation: indeed if we set $(\mathcal{H} p)^{M}=\diamond p$ then we cannot express an equivalent of $(\vdash p)^{\perp}$ in the language $\mathcal{L}^{H}$, and similarly there is no equivalent of $(\mathcal{H} p)^{\perp}$ in the language $\mathcal{L}^{A}$. Hence the meta-theoretic operation ()$^{\perp}$ becomes a partial function.

\subsection{Examples}

We give here an example of derivation in the sequent calculus AH-G1, whose axioms and rules are in Appendix, Section 9.

For $A$ be assertive and $C$ hypothetical we use the following abbreviations:

- $\square C:=\sim\left((C)^{\perp}\right)$ and symmetrically

- $\diamond A:=\frown\left((A)^{\perp}\right)$.

Proposition 4. The following sequents are provable in AH-G1.

(i) $A^{\perp \perp} ; \Rightarrow A$; and $A ; \Rightarrow A^{\perp \perp}$; and symmetrically; $C \Rightarrow ; C^{\perp \perp}$ and $; C^{\perp \perp} \Rightarrow ; C$.

(ii) $A ; \Rightarrow \triangleright \odot A$; and ; $\odot \odot C \Rightarrow$; $C$.

(iii) $M \supset \square C \Rightarrow \square\left(M^{\perp} \curlyvee C\right)$ and $\square\left(M^{\perp} \curlyvee C\right)$; $\Rightarrow$; $M \triangleright C$.

Proof. (i) and (iii) are left as an exercise. 


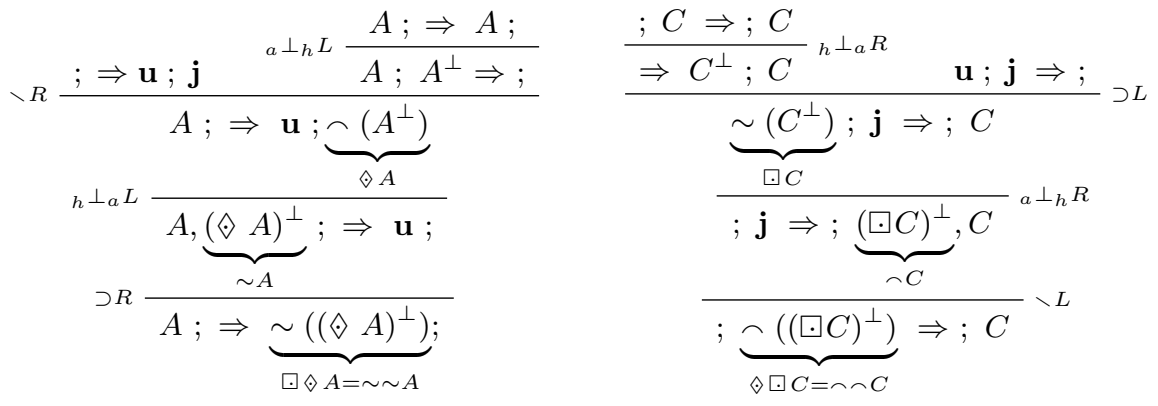

By applying meta-theoretic dualities the sequents in $(i i)$ are equivalent to

$$
\text { (ii) } A \Rightarrow \sim \sim A \text { and } ; \frown C \Rightarrow ; C
$$

Moreover the sequents in $($ iii $)$ are simplified as the equivalence

$$
\sim\left(M \cap C^{\perp}\right) \Leftrightarrow\left(M \supset \sim\left(C^{\perp}\right) .\right.
$$

In [10] we were interested in the notion of chirality proposed by Paul-André Melliès [34] and in the following conjecture:

\section{Bi-intuitionistic logic AHL constitutes a dialogue chirality.}

With the present definition (4) in Section 4.2 the conjecture is plausible. We give in the Appendix, Section 9, the main definition of chirality and the considerations concerning Melliès conjecture.

\section{Expectations and Conjectures.}

In [8] two new "illocutionary forces" were introduced in the language $\mathcal{L}^{A H}$ of assertions and hypotheses: expectations $\mathcal{E} \alpha$ and conjectures $\mathcal{C} \alpha$ with the following modal translation:

$$
(\mathcal{E} \alpha)^{M}:=\square \diamond \alpha \quad \text { and } \quad(\mathcal{C} \alpha)^{M}:=\diamond \square \alpha
$$

The intuitive explanation of these illocutionary forces is supported and in part explained by the modal translation and its Kripke semantics:

- the expectation $\mathcal{E} \alpha$ that $\alpha$ is true is the assertion that the truth of $\alpha$ is epistemically possible or in any situation a situation can be reached where $\alpha$ is true.

- The conjecture $\mathcal{C} \alpha$ that $\alpha$ is true is the hypothesis that the truth of $\alpha$ is epistemically necessary or a situation can be reached where $\alpha$ is true in all situations resulting from it.

As above, we consider only the case of $\alpha$ atomic and we write $\mathcal{E} p^{*}$ and $\mathcal{C} p$ to make sure that expectations and conjectures are understood as dual. 


\subsection{Expectations: classical logic in intuitionistic pragmatics.}

Let $\mathcal{L}^{E}$ be the language defined by the grammar.

$$
E, F \quad:=\mathcal{E} p^{*}|\curlyvee| E \supset F|E \cap F| \sim E
$$

and let $\mathbf{E L}$ be the set of all formulas $E$ in the language $\mathcal{L}^{E}$ whose $\mathbf{S} \mathbf{4}$ translation is valid.

Proposition 5. The theory EL (logic of expectations) is closed under the double negation rule, i.e., $\sim E \Rightarrow E$ is a valid axiom of $\mathbf{E L}$.

It is an exercise in the modal logic $\mathbf{S} \mathbf{4}$ to prove the proposition by induction on the definition of $\mathcal{L}^{E}$ formulas.

On the other hand, if we extend $\mathcal{L}^{E}$ with intuitionistic disjunction $(\cup)$, then the inductive step for disjunction fails:

$$
\left(\sim \sim\left(\mathcal{E} p^{*} \cup \mathcal{E} q^{*}\right)\right) \not \equiv\left(\sim \sim \mathcal{E} p^{*}\right) \cup\left(\sim \sim \mathcal{E} q^{*}\right) .
$$

Remark 2. It is known [52] that a direct translation $\left(A^{m}\right)$ of the full language of (propositional) classical logic in classical $\mathbf{S} \mathbf{4}$ can be given mapping $p \mapsto$ $\square \diamond p$; but here, crucially, we need the translation $(A \vee B)^{m}=\square \diamond\left(A^{m} \vee B^{m}\right)$. On the other hand, excluding disjunction from the classical (propositional) language we still obtain the $A^{m}$ modal translation from the modal translation of the connectives of intuitionistic logic, starting from the map $p \mapsto \square \diamond p$ for the atoms ${ }^{3}$. Thus we have given a pragmatic interpretation of a classical result.

A completely symmetric discussion applies to conjectures, where the language of conjectures is closed under the following rule which is co-intuitionistically invalid:

$$
\mathcal{C} p \Rightarrow \frown \mathcal{C} p
$$

Remark 3. Consider the bi-intuitionistic language $\mathcal{L}^{A H}$ Notice that $\left(\mathcal{E} p^{*}\right)^{M} \equiv$ $\left(\sim\left(\mathcal{H} p^{*}\right)^{\perp}\right)^{M}:$

$$
\left(\sim\left(\mathcal{H} p^{*}\right)^{\perp}\right)^{M}=\square \neg \neg \diamond p^{*}=\square \diamond p^{*}
$$

Similarly, $(\mathcal{C} p)^{M} \equiv\left(\frown(\vdash p)^{\perp}\right)^{M}$ :

$$
\left(\frown(\vdash p)^{\perp}\right)^{M}=\diamond \neg \neg \square p=\diamond \square p .
$$

This fact can be generalized. Let $A$ be assertive and $C$ hypothetical. We write

- Let $\square C:=\sim\left((C)^{\perp}\right)$. Then $(\square C)^{M}=\square \triangleright C^{M}$. Symmetrically

- Let $\odot A:=\frown\left((A)^{\perp}\right)$. Then $(\diamond A)^{M}=\diamond \square A^{M}$.

${ }^{3}$ Grigori Mints showed us the connection between our work and the classical translation $\square \diamond[52]$. 


\section{Part II Co-intuitionistic pragmatics.}

In Part II we assume the usual definition hypotheses $\mathcal{H} p$, but the same remarks apply to for negative hypotheses

\subsection{Justification conditions and semantic values for hypotheses.}

How are justification conditions and semantic values assigned to hypothetical expressions? Answering this question is difficult. We want to extend the BHK interpretation to a co-intuitionistic logic of hypotheses. In the case of intuitionistic assertive $A$, a justification for $A$ is conclusive evidence for $A$; here what counts as conclusive evidence is relative to the standards of scientific disciplines, in particular, in mathematics we need a proof of $A$. To characterise justification conditions for hypotheses we may use use legal terminology and say that a justification for $\mathcal{H} p$ is a "scintilla of evidence" that $p$ may be true. ${ }^{4}$ It is quite evident that the justification conditions of the expression $\mathcal{H} p$ are problematic and constitutes a weak spot for the generalisation of the BHK interpretation.

There are at least two approaches: one is to say that if it is compatible with the context of our knowledge to make the hypothesis $C$, then the simple awareness of this generic compatibility must count as evidence for $C$. Another approach is to say that, on the contrary, some circumstances giving positive confirmation for the truth of $C$ are required. Let us consider in particular the Example 1 in Section 2.1.

Poincaré's statement is certainly compatible with our present knowledge, although it is beyond our means to put it to test; but exactly the same can be said of its negation. Hence the only "scintilla of evidence " for it and for its negation is a mere realization of compatibility. In general any proposition $p$ a classical logician may consider as meaningful and capable of a semantic value can also be tested for compatibility with our present knowledge; if the result of this test is positive, then it constitutes evidence for $\mathcal{H} p$. In a classical theory of meaning the statement may be regarded as meaningful and its compatibility with our present knowledge may justify considering it as an abstract hypothesis, albeit not a viable scientific hypothesis. On the other hand, there is no doubt that for an intuitionistic viewpoint like Dummett's the statement ought to be regarded as meaningless and there can be no spark of evidence for a meaningless statement. Moreover it seems that in the approach "hypothetical evidence as confirmation" we are exploring, a "scintilla of evidence" for a

\footnotetext{
${ }^{4}$ In the theory of argumentation for legal reasoning, six proof standards have been identified: no evidence at all, scintilla of evidence, preponderance of evidence, clear and convincing evidence, beyond reasonable doubt and dialectical validity.[30, 17] Let's identify "dialectical validity" with conclusive evidence, justifying assertions, and "scintilla of evidence", as the standard justifying hypotheses: the other three standards are defined through probabilities.
} 
hypothesis cannot be just an abstract test of compatibility, but does require some positive evidence.

\subsection{Hypothetical contexts and connectives.}

An intuitionistically acceptable explanation of hypothetical co-intuitionistic logic is in Dummett and Prawitz's meaning as use approach [23, 43]. Such an approach usually gives an account of how the justification condition for molecular expressions depend on the justification condition of the immediate components according to the meaning of their main connective. Here the meaning of a connective may be explained according to $(a)$ an introduction rule in Natural Deduction [a right rule in the sequent calculus] or $(b)$ according to an elimination rule [a left rule] [43]. In case $(a)$ one shows how the justification conditions of a molecular formula are determined from the justification conditions of its component formulas; in case $(b)$ one shows how the immediate consequences of a molecular formula are determined from the immediate consequences of its component formulas. Then, roughly speaking, the justification of an introduction-elimination pair [a right-left pair] follows from the inversion principle [40], by showing that nothing is derivable after a normalization step [a symmetric cut elimination step] that was not derivable before.

We discussed in some detail a meaning as use explanation of co-intuitionistic connectives in [10], section 2.1 and we shall not repeat it here. In our view, however, such an an explanation must also explain the justification conditions of elementary formulas, as done here in section (6.1), but also the meaning of the co-intuitionistic consequence relation

$$
C \Rightarrow D_{1}, \ldots, D_{n}
$$

To explain the consequence relation in (8), two approaches are available, which were discussed also in [10]. One, which we sketch here, explains how given evidence $x$ for the hypothesis $C$ we obtain "parcels of hypothetical evidence" $t_{1}, \ldots, t_{n}$ for $D_{1}, \ldots, D_{n}$. More precisely, following the BHK interpretation. we may say that an effective interpretation of (8) is as follows:

H.1: "there is a method $F$ transforming sufficient evidence for justifying the hypothesis $C$ into sufficient evidence for justifying (the disjunction of) the hypotheses $D_{1}, \ldots, D_{n}$.

The second approach is to explain how given refutations for $D_{1}, \ldots, D_{n}$ one constructs a refutation of $C$ :

H.2: "there is a method $F^{o p}$ to transform evidence refuting all the hypotheses $D_{1}, \ldots, D_{n}$ into evidence refuting the hypothesis $C$.

The latter approach fits in well with an interesting account of co-intuitionism by Y. Shramko [49]: co-intuitionistic sentences are interpreted as statements 
that have not yet been refuted. Following Popper, scientific laws formalizable as universally quantified statements cannot be conclusively confirmed, but can and must in principle be falsifiable; hence co-intuitionism is the logic of scientific research. In the second approach it is conclusive evidence that is considered and transformed; in mathematics, refuting a hypothesis $C$ amounts to prove a theorem $C^{\perp}$. We shall explore the first approach, assuming that we know what sufficient grounds for hypotheses are.

We give an informal account of the second approach, formalized as a "calculus of co-intuitionistic coroutines" as done in the papers [8, 9, 13], adapting an idea by Crolard [19]. Thus we have a typed computational context of justification

$$
x: C \Rightarrow \mathcal{S}_{x}: \Delta
$$

where in $\mathcal{S}_{x}=t_{1}, \ldots, t_{n}$ consists of separate "parcels of evidence" for the hypotheses $D_{1}, \ldots, D_{n}=\Delta$. Essential to the co-intuitionistic nature of the calculus is that $C$ is the only open assumption in the context, thus that $x$ is the one and only free variable occurring in $\mathcal{S}_{x}$. We may think of a distributed system, where each term $t_{i}: D_{i}$ has a location within a context connected by $x .^{5}$

We focus on subtraction, as the treatment of the other connectives is more familiar. We assign terms of our calculus to the following sequent calculus rules and we explain the operational semantics of our calculus which reflects adequately the pragmatic meaning of logical operations.

$$
\frac{H \Rightarrow \Gamma, C \quad D \Rightarrow \Delta}{H \Rightarrow \Gamma, C \backslash D, \Delta} \backslash \mathrm{R} \quad \frac{C \Rightarrow D, \Upsilon}{C \backslash D \Rightarrow \Upsilon} \backslash \mathrm{L}
$$

with reduction rule

$$
\begin{aligned}
& \frac{H \Rightarrow \Gamma, C \quad D \Rightarrow \Delta}{H \Rightarrow \Gamma, C \backslash D, \Delta} \backslash \mathrm{R} \quad \frac{C \Rightarrow D, \Upsilon}{C \backslash D \Rightarrow \Upsilon} \backslash \mathrm{L} \\
& \text { reduces to }
\end{aligned}
$$

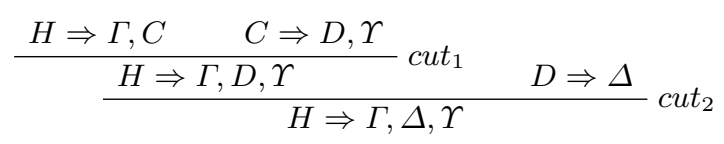

Suppose we have a context $\mathcal{S}_{z}$ indexed with $z$. We write it in the form of a sequent

$$
z: C \Rightarrow \ell: D, \mathcal{S}_{z}: \Upsilon
$$

where $\ell$ is a list of zero or more terms giving justifications of $D$. We need lists because there is a rule of contraction right in the co-intuitionistic calculus.

\footnotetext{
${ }^{5}$ We speak of a distributed system as a promising metaphor: no such implementation of the calculus has been done. Without using spatial terms one can say "clearly distinct from", rather than instead of "being away from".
} 
Moreover suppose we have also a context consisting only of the variable $v$ : $C \backslash D$ To have a justification $v$ of $C \backslash D$ means to be able to extract from it a justification $\mathbf{z}(v)$ for $C$ and also to set aside the part of the derivation yielding a justification $\ell$ of $D$ from the justification $z$ of $C$ : we use a term postpone $(z \mapsto \ell, v)$ for this purpose. Here "setting aside" $\ell$ is the operational consequence of the inconsistency between $v: C \backslash D$ and the evidence $\ell: D{ }^{6}$ Finally, in $\mathcal{S}_{z}$ the variable $z$ is now substituted by $\mathrm{z}(v)$. Thus the subtraction left rule as follows:

$$
\frac{z: C \Rightarrow \ell: D, \mathcal{S}_{z}: \Upsilon}{v: C \backslash D \Rightarrow \text { postpone }(z \mapsto \ell, v): \bullet, \mathcal{S}_{z(v)}: \Upsilon} \backslash \mathrm{L}
$$

In the subtraction right rule we have an operation make-coroutine connecting two computational contexts, namely,

$$
x: H \Rightarrow \mathcal{S}_{x}: \Gamma, t: C \quad y: D \Rightarrow \mathcal{S}_{y}: \Delta .
$$

We can merge the two contexts into one, still depending on $x: H$, by deriving (i) either a justification of $C \backslash D(i i)$ or a justification of $D$ given that of $C$. In case $(i)$ we write make-coroutine $(t, \mathrm{y}): C \backslash D$; in case $(i i)$ we have a justification $\mathrm{y}(t): D$.

Now we substitute $\mathrm{y}(t)$ for $y$ in $\mathcal{S}_{y}: \Delta$, expressing the dependency of the context $\mathcal{S}_{\mathrm{y}(t)}: \Delta$ from $t$. This is clearly distinct from $\mathcal{S}_{x}$, being indexed with $\mathrm{y}(t)$. Notice however that $x$ occurs free in $t$; hence $x$ occurs everywhere in $\mathcal{S}_{\mathrm{y}(t)}$. The $C \backslash D$ right rule is as follows:

$$
\frac{x: H \Rightarrow \mathcal{S}_{x}: \Gamma, t: C \quad y: D \Rightarrow \mathcal{S}_{y}: \Delta}{x: H \Rightarrow \mathcal{S}_{x}: \Gamma, \text { make-coroutine }(t, \mathrm{y}): C \backslash D, \mathcal{S}_{\mathrm{y}(t)}: \Upsilon} \backslash \mathrm{R}
$$

Now consider a cut where the left and right cut-formula $C \backslash D$ has been introduced by the right and left rules, as in (11) and (10), respectively. Then a justification for $C \backslash D$ of the form make-coroutine is substituted for $y$ in postpone $(x \mapsto \ell, y)$. The upshot is that when eliminating the cut, the "postponed" computation $\ell$ can be used to "fill the jump" from $C$ to $D$ in (11).

$$
\frac{x: H \Rightarrow \mathcal{S}_{x}: \Gamma, t: C \quad z: C \Rightarrow \ell: D, \mathcal{S}_{z}: \Delta}{\frac{x: H \Rightarrow \mathcal{S}_{x}: \Gamma, \ell^{\prime}: D, \mathcal{S}_{t}: \Delta}{x: H \Rightarrow \mathcal{S}_{x}: \Gamma, \mathcal{S}_{t}: \Delta, \mathcal{S}_{\ell^{\prime}}: \Upsilon} y: D \Rightarrow \mathcal{S}_{y}: \Upsilon}
$$

where $\mathcal{S}_{t}=_{d f} \mathcal{S}_{z}\{z:=t\}, \ell^{\prime}={ }_{d f} \ell\{z:=t\}$ and $\mathcal{S}_{\ell^{\prime}}$ is a list of contexts resulting from repeatedly substituting each term $j \in \ell^{\prime}$ for $z$ in $\mathcal{S}_{z}$.

Summing up, the operational interpretation of the postpone operation assigned to the subtraction left rule is essentially in the act of removing from the

\footnotetext{
${ }^{6}$ The postpone term is not assigned to a formula: we may assign it to a symbol • which is not a type and thus cannot occur as a sub-formula of other formulas.
} 
current space of justification the derivation of a justification for $D$ from a justification for $C$, asauming $D$ to be incompatible with $C$. This is not so far from the meaning as use interpretation of the left subtraction rule, which defines the meaning of the connective of subtraction in Dummett and Prawitz's interpretation. We see here that co-intutionistic dialectic creates new hypotheses in the subtraction right and removes some of them in subtraction left and does not reach the logical level of assertions (except perhaps in the case where all consequences of an assumption $v: H$ have been removed). Yet this calculus is in some sense "symmetric" to the simply typed $\lambda$-calculus with pairing and projections and can simulate $\lambda$ computations by cut-elimination [8].

\section{The linear case.}

Roughly speaking, the linear calculus of co-intuitionistic coroutines is defined by the restriction that all operators are applied to terms and not lists of terms. In particular, we have postpone $(x \mapsto t, u)$ instead of postpone $(x \mapsto \ell, u)$.

Co-intuitionistic logic is a very static model of computation. Coroutines in the sense of Tristan Crolard [19] are defined in the very rich environment of the $\lambda \mu$-calculus, which gives a computational interpretation of classical logic. Here we do not exit from co-intuitionistic dialectic. An interesting feature of this calculus is in the treatment of parallel composition in its interaction with the operators.

Some applications of co-intuitionistic logic to models of computation were investigated in the linear calculus.

- Our calculus of co-routines is essentially based on contexts $\mathcal{S}_{x}$; these consist of the parallel composition of term, induced and modified (among other) by the make-coroutine and postpone operations. To explore its connection with parallel and distributed computing a comparison with the $\pi$-calculus and with membrane computing ( $\lambda P$-systems) was then sketched in [13].

- A categorical model of linear co-intuitionistic logic with exponentials was given by the first author in [9], based on G. Bierman's thesis [16]. There the calculus of coroutines was used as a term assignment for the categorical construction.

\subsection{Linear co-intuitionism}

We give here precise definitions for the linear calculus of co-intitionistic coroutines, typed in linear co-intuitionistic logic with subtraction, par $(\wp)$ and $\perp$, the identity of disjunction.

Definition 5. We are given a countable set of free variables (denoted by $x$, $y, z \ldots$ ), and a countable set of unary functions (denoted by $\mathrm{x}, \mathrm{y}, \mathrm{z}, \ldots$ ). The 
terms of our calculus, denoted by $R$, are either $m$-terms, denoted by $M, N$, or $p$-terms, denoted by $P$.

(i) Terms, $m$-terms and p-terms are defined by the following grammar.

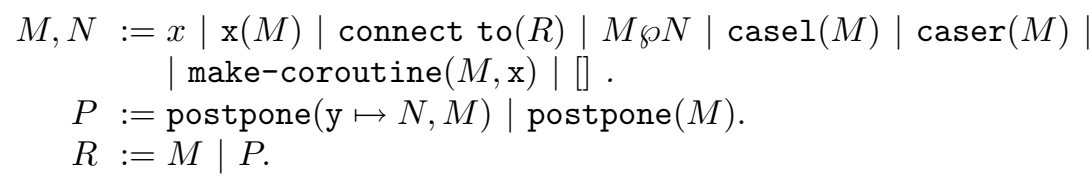

We usually abbreviate "make - coroutine" as "mkc" and "postpone" as "postp". We often write $\bar{P}$ for a list $P_{1}, \ldots, P_{m}$, and similarly $\bar{M}$ for $M_{1}, \ldots, M_{n}$. "[]" is the empty term (nil).

Definition 6. (i) The free variables $F V(M)$ in a term $R$ are defined thus:

$$
\begin{aligned}
& F V([])=\emptyset \\
& F V(x)=\{x\} \\
& F V(\mathrm{x}(M))=F V(M) \\
& F V(M \wp N)=F V(M) \cup F V(N) \\
& F V(\operatorname{connect} \operatorname{to}(R))=F V(R) \\
& F V(M)=F V(M) \\
& F V(\operatorname{maser}(M, \mathrm{x}))=F V(M) \\
& F V(\operatorname{casel}(M))=F(\min (M) \backslash\{x\}) \cup F V(M) . \\
& F V(\operatorname{postp}(\mathrm{x} \mapsto N, M))=(F V(N) \backslash \\
& F V(\operatorname{postp}(M))=F V(M) .
\end{aligned}
$$

Definition 7. (i) Let $\|$ be a binary operation on terms (parallel composition) which is associative, commutative and has the empty term [] as the identity. Terms generated by (zero or more) applications of parallel composition are called contexts. Thus contexts are generated by the following grammar:

$$
C:=R \mid(C \| R)
$$

modulo the structural congruences

(i) $\quad R_{0}\left\|\left(R_{1} \| R 2\right) \equiv\left(R_{0} \| R_{1}\right)\right\| R 2$,

(ii) $\quad R_{0}\left\|R_{1} \equiv R_{1}\right\| R_{0}$,

(iii) $\quad R_{0} \|[] \equiv R_{0}$,

(iv) $\quad C_{0}\|R\| C_{1} \equiv C_{0}\left\|R^{\prime}\right\| C_{1}$ if $R \equiv R^{\prime}$.

Let $R_{1}\|\ldots\| R_{k}$ be a context, where all $R_{i}$ are non-null, $i \leq k$. Notice that the notation is well-defined by generalized associativity. We write $\mathcal{S}_{\bar{x}}: R_{1}\|\ldots\| R_{k}$ if all free variables occurring in $R_{1}, \ldots, R_{k}$ are in the list $\bar{x}$.

Definition 8. An expression $\mathcal{S}_{x}: R_{1}\|\ldots\| R_{k}$ is a (correct) computational context if it satisfies the following axioms.

1. Each term in the set $\left\{R_{1}, \ldots, R_{k}\right\}$ contains $x$ and no other free variable.

2. In every term of the form postp $(\mathrm{y} \mapsto N, M)$ the term $N$ contains a free variable $y$ with $y \notin F V(M)$ and no other free variable. 
A computational context can be denoted by listing the non-empty terms it contains: $\mathcal{S}_{x}=P_{1}, \ldots, P_{m} \mid M_{1}, \ldots, M_{n}$. (The separation "|" between $p$-terms and $m$-terms is only a notational convenience in view of typing).

Remark 4. By axiom 1 the relevant components of a computational context are uniquely identified. Axiom 2 is analogue to the acyclicity condition in proof nets for linear logic.

There is a standard, but combersome notion of $\alpha$-equivalence which we skip (see [9]).

Note 1. (i) Consider the terms make - coroutine and binary postpone. They are binders acting on a whole computational context, rather than on a delimited scope within a single term. We may call their action remote binding; it is expressed by a substitution of some $m$-term $\mathrm{x}(M)$ for the free variable $x$ throughout a computational context $\mathcal{S}_{x}$. One could express remote binding by a more familiar notation, as in the $\lambda$-calculus or in the $\pi$-calculus; but then in the typed case one could not assign a separate $m$-term to each formula in the succedent. At best, one could assign an "access port" to a unique term assigned to the whole sequent, as in the translations of linear logic into the $\pi$-calculus (see [13]). A motivation here is to create a "distributed calculus" for our "multiple conclusion" logical deductive system.

(ii) The $p$-term binary postpone is also a local binder of the free variable occurring in its argument. Indeed in a term postpone $(\mathrm{y} \mapsto N, M)$ a free variable $y$ occurring in $N$ becomes locally bound; then $y$ is replaced by $\mathrm{y}(M)$ in the computational context, to express remote binding. A more complete notation of our p-terms would be postpone $(y \mapsto N, M)$ with y for $y$, explicitly establishing the connection between the locally bound variable and its corresponding unary function.

Definition 9. Substitution of a term $M$ for a free variable $x$ in a term $R$ is defined as follows:

$$
\begin{aligned}
x[x:=M]=M, & y[x:=M]=y \text { if } x \neq y ; \\
\operatorname{connect~to}(N)[x:=M] & =\operatorname{connect} \operatorname{to}(N[x:=M]) \\
\operatorname{postp}(N)[x:=M] & =\operatorname{postp}(N[x:=M]) \\
\mathrm{y}(N)[x:=M] & =\mathrm{y}(N[x:=M]) ; \\
\left(N_{0} \wp N_{1}\right)[x:=M] & =\left(N_{0}[x:=M]\right) \wp\left(N_{1}[x:=M]\right) \\
\operatorname{casel}(N)[x:=M] & =\operatorname{casel}(N[x:=M]), \\
\operatorname{caser}(N)[x:=M] & =\operatorname{caser}(N[x:=M]) ; \\
\operatorname{mkc}(N, \mathrm{y})[x:=M] & =\operatorname{mkc}(N[x:=M], \mathrm{y}), \\
\operatorname{postp}\left(\mathrm{y} \mapsto\left(N_{1}\right), N_{0}\right)[x:=M] & =\operatorname{postp}\left(\mathrm{y} \mapsto\left(N_{1}[x:=M]\right), N_{0}[x:=M]\right) .
\end{aligned}
$$

Proposition 6. $(i) \mathcal{S}_{x}=x$ and $\mathcal{S}_{y}=\operatorname{postp}(y)$ are computational contexts.

Let $\mathcal{S}_{x}=R_{1}\|\ldots\| R_{m} \| M$ and $\mathcal{S}_{y}=R_{m+1}\|\ldots\| R_{m+n}$ be computational contexts. We write $\mathcal{S}_{y}[y:=N]$ for $R_{m+1}[y:=N]\|\ldots\| R_{m+n}[y:=N]$. Then 
(ii) $\mathcal{S}_{x}^{\prime}=R_{1}\|\ldots\| R_{m} \|\left(\mathcal{S}_{y}[y:=M]\right)$ is a computational context (substitution);

(iii) $\mathcal{S}_{x}^{\prime}=R_{1}\|\ldots\| R_{m}\|\operatorname{mkc}(M, \mathrm{y})\| \mathcal{S}_{y}[y:=\mathrm{y}(M)]$ is a computational context (make coroutine);

(iv) Let $\mathcal{S}_{x}=R_{1}\|\ldots\| R_{m}$ and $\mathcal{S}_{y}=R_{m+1}\|\ldots\| R_{m+n}$ be computational contexts. Then

$$
\mathcal{S}_{z}=\mathcal{S}_{x}[x:=\operatorname{casel}(z)] \| \mathcal{S}_{y}[y:=\operatorname{caser}(z)]
$$

is a computational context (cases);

(v) Let $\mathcal{S}_{x}=R_{1}\|\ldots\| R_{m} \| M$ be a computational context. Then

$$
\mathcal{S}_{y}=\operatorname{postp}(x \mapsto M, y)\left\|R_{1}\right\| \ldots \| R_{m}
$$

is a computational context (postpone);

(vi) Let $\mathcal{S}_{x}=R_{1}\|\ldots\| M_{i}\left\|M_{i+1}\right\| \ldots \| R_{m}$ be a computational context. Then $\left.\mathcal{S}_{x}^{\prime}=R_{1}\|\ldots\|\left(M_{i} \wp M_{i+1}\right)\|\ldots\| R_{m}\right\}$ is a computational context (par).

In all cases the proposition is proved simply by checking that the resulting set of terms contains exactly one free variable.

The operation $\beta$-reduction transforms a computational context $\mathcal{S}_{x}$ into a computational context $\mathcal{S}_{x}^{\prime}$. It may be either local, affecting only the redex itself, or a global operation with side-effects on parts of $\mathcal{S}_{x}$ mainly, relabelling the terms that express binding by make - coroutine, postpone or store.

Definition 10. $\beta$-reduction of a redex $\mathcal{R}$ ed in a computational context $\mathcal{S}_{x}$ is defined as follows.

(i) If Red is a m-term $N$ of the following form, then the reduction is local and consists of the rewriting $N \rightsquigarrow \beta N^{\prime}$ in $\mathcal{S}_{x}$ as follows:

$$
\begin{gathered}
\operatorname{postp}(\operatorname{connect} \text { to }(R)) \rightsquigarrow \beta[] . \\
\text { casel }\left(N_{\left.0 \wp N_{1}\right) \rightsquigarrow \beta} N_{0} ; \quad \operatorname{caser}\left(N_{0} \wp N_{1}\right) \rightsquigarrow \beta\right.
\end{gathered}
$$

If the principal operator of $\mathcal{R}$ ed is a binary postpone, then the reduction is global and consists of the following rewriting.

(ii) If $\mathcal{R}$ ed has the form $\operatorname{post} \mathrm{p}(\mathrm{z} \mapsto N, \operatorname{mkc}(M, \mathrm{y}))$, then $\mathcal{S}_{v}$ is partitioned as

$$
\mathcal{S}_{v}=\mathcal{R} e d \cup \mathcal{S}_{v y z}[y:=\mathrm{y}(M), z:=\mathrm{z}(\operatorname{mkc}(M, \mathrm{y}))]
$$

(a simultaneous substitution of $\mathrm{y}(M)$ for $y$ and of $\mathrm{z}(\operatorname{mkc}(M, \mathrm{y}))$ for $z$ in $\left.\mathcal{S}_{v y z}\right)$. Then a reduction of $\mathcal{R}$ ed transforms the computational context as follows:

$$
\mathcal{S}_{v}=\mathcal{S}_{v y z}[y:=N[z:=M], z:=M] .
$$

The use of computational contexts in the decoration of sequent-style Natural Deduction derivations in co-intuitionistic logic is illustrated in Table 1. 


$$
\begin{aligned}
& \begin{array}{c}
\text { axiom } \\
x: A \triangleright \mid x: A
\end{array} \frac{v: E \triangleright \bar{P}|\bar{M}: \Gamma, M: A \quad x: A \triangleright \bar{Q}| \bar{N}: \Delta}{x: E \triangleright \bar{P}, \bar{Q}^{\prime}[x:=M] \mid \bar{M}: \Gamma, \bar{N}[x:=M]: \Delta} \\
& \perp \text {-intro } \\
& \frac{x: E \triangleright \bar{P} \mid \bar{M}: \Gamma \quad(R \in \bar{P} \cup \bar{M})}{x: E \triangleright \bar{P} \mid \bar{M}: \Gamma, \text { connect to }(R): \perp} \quad \begin{array}{l}
\perp \text {-elim } \\
\quad x \operatorname{postp}(x) \mid
\end{array} \\
& \text { We write } \bar{Q}^{\prime} \text { for } \bar{Q}[x:=\mathrm{x}(M)] \text { and } \bar{N}^{\prime} \text { for } \bar{N}[x:=\mathrm{x}(M)] \\
& \backslash \text {-intro } \\
& \begin{array}{c}
v: E \triangleright \bar{P}|\bar{M}: \Gamma, M: C \quad x: D \triangleright \bar{Q}| \bar{N}: \Delta \\
v: E \triangleright \bar{P}, \bar{Q}^{\prime} \mid \bar{M}: \Gamma, \bar{N}^{\prime}: \Delta, \operatorname{mkc}(M, \mathrm{x}): C \backslash D
\end{array} \\
& \backslash \text {-elim } \\
& \frac{z: E \triangleright \bar{P}|\bar{M}: \Gamma, M: C \backslash D \quad x: C \triangleright \bar{Q}| N: D, \bar{N}: \Delta}{z: E \triangleright \bar{P}, \bar{Q}^{\prime}, \operatorname{postp}(x \mapsto M, z) \mid \bar{M}: \Gamma, \bar{N}^{\prime}: \Delta} \\
& \wp \text {-intro } \\
& \frac{x: E \triangleright \bar{P} \mid \bar{M}: \Gamma, M_{0}: C_{0}, M_{1}: C_{1}}{x: E \triangleright \bar{P} \mid \bar{M}: \Gamma, M_{0} \wp M_{1}: C_{0} \wp C_{1}} \\
& \wp \text {-elim } \\
& z: E \triangleright \bar{Q} \mid \bar{N}: \Delta, N: C_{0} \wp C_{1} \\
& \frac{x_{0}: C_{0} \triangleright \bar{P}_{0}\left|\bar{M}_{0}: \Gamma_{0} \quad x_{1}: C_{1} \triangleright \bar{P}_{1}\right| \bar{M}_{1}: \Gamma_{1}}{z: E \triangleright \bar{Q}, \bar{P}_{0}^{\prime}, \bar{P}_{1}^{\prime} \mid \bar{N}: \Delta, \bar{M}_{0}^{\prime}: \Gamma_{0}, \bar{M}_{1}^{\prime}: \Gamma_{1}} \\
& \text { where } \bar{P}_{0}^{\prime}=\bar{P}\left[x_{0}:=\operatorname{casel}(z)\right], \bar{M}_{0}^{\prime}=\bar{M}_{0}\left[x_{0}:=\operatorname{casel}(z)\right] \text {, } \\
& \bar{P}_{1}^{\prime}=\bar{P}_{1}\left[x_{0}:=\operatorname{caser}(z)\right], \bar{M}_{1}^{\prime}=\bar{M}_{1}\left[x_{0}:=\operatorname{caser}(z)\right]
\end{aligned}
$$

Table 1. Decorated Natural Deduction for multiplicative co-ILL

\subsection{Examples of computational contexts.}

1. The simplest example is that of a $p$-term postpone $(x)$ alone.

$$
\mathrm{S}_{x}: \quad \text { postpone }(x) \text {. }
$$

Its typing is given by the axiom $x: \perp \triangleright \operatorname{postp}(x) \mid$.

2. Let

$$
\mathcal{S}_{x}=\operatorname{postp}(x) \| \text { connect to }(\operatorname{postp}(x)) \| \text { connect to }(\operatorname{postp}(x)) .
$$


It can be regarded as an expansion of $\mathcal{S}_{x}^{\prime}: x \|$ connect to $(x)$. Their typings are as follows:

$$
x: \perp \triangleright \operatorname{postp}(x) \mid \text { connect to }(\operatorname{postp}(x)): \perp, \text { connect to }(\operatorname{postp}(x)): \perp
$$

and

$$
x: \perp \triangleright \mid x: \perp \text {, connect to }(x): \perp \text {. }
$$

3. Let $\mathcal{S}_{y}$ be $\operatorname{postp}(y)$ and $\mathbf{S}_{x}=x \|$ connect to $(x)$. We obtain a new computational context by parallel composing $x$ with the result of substituting connect to $(x)$ for $y$ in $\mathcal{S}_{y}$ :

$$
\mathrm{S}_{x}^{\prime}=x \| \text { postp }(\text { connect to }(x))
$$

which $\beta$-reduces to $\mathbf{S}_{x}^{\prime \prime}: x \|[]$. The typed versions are as follows:

$$
x: \perp \triangleright \text { postp }(\text { connect to }(x)) \mid x: \perp \text { reduces to } x: \perp \triangleright x: \perp \text {. }
$$

4. Let $\mathcal{S}_{x}=\operatorname{mkc}(x, \mathrm{y}) \| \mathrm{y}(x)$ and let $\mathcal{S}_{v}=\operatorname{postp}(z \mapsto z, v)$. Then substituting $\operatorname{mkc}(x, \mathrm{y})$ for $v$ in $\mathcal{S}_{v}$ we obtain a new computational context

$$
\mathcal{S}^{\prime}{ }_{x}=\operatorname{postp}(z \mapsto z, \operatorname{mkc}(x, \mathrm{y})) \| \mathrm{y}(x)
$$

which $\beta$-reduces to

$$
\mathcal{S}_{x}^{\prime \prime}=x
$$

A typing of $\mathcal{S}_{x}^{\prime}$ is

$$
\frac{\frac{x: C \triangleright x: C \quad y: C \triangleright y: C}{x: C \triangleright \operatorname{mkc}(x, \mathrm{y}): C \backslash C, \mathrm{y}(x): C} \quad z: C \triangleright z: C}{x: C \triangleright \operatorname{postp}(z \mapsto z, \operatorname{mkc}(x, \mathrm{y}) \mid \mathrm{y}(x): C}
$$

The $\beta$-reduction amounts to performing two substitutions, first of $x$ for $z$ and then of $x$ for $y$ :

$$
\frac{x: C \triangleright x: C \quad z: C \triangleright z: C}{\frac{x: C \triangleright x: C}{x: C \triangleright x: C} y: C \triangleright y: C}
$$

In the setting of a term calculus for co-intuitionistic logic parallel composition is an operation on the terms of the multiple-conclusion logical context, inductively defined form primitive constructors and destructors operators to form a computational context. Such constructions make sense also in an untyped setting, where computational contexts are subject only to a mild constraint, the presence of exactly one free variable in them.

\subsection{A probabilistic interpretation.}

The sequent calculus with term-assignment informally described in Section 6.2 allows us to establish a connection with probabilistic interpretations of hypotheses. The following result is from [9]. 
In our setting co-intuitionistic logic admits a simple probabilistic interpretation which fits well in the view of co-intuitionism as a logic of hypotheses. Indeed if co-intuitionistic logic is about the justification properties of hypotheses, which we now assess in a probabilistic setting, then the co-intuitionistic consequence relation must preserve probability assignments from the premises to the conclusions; a term calculus for such a logic must allow us to compute probabilities and verify the preservation property. We sketch our result only for the multiplicative linear fragment, i.e., for typing derivations in the linear system with subtraction only.

Definition 11. To the typed terms of linear co-intuitionistic logic we assign events in a probabilistic setting. We write $\overline{\mathbf{C}}, \mathbf{C} \cap \mathbf{D}$ and $\mathbf{C} \cup \mathbf{D}$ for complementation, intersection and union between events; there is an impossible event $\emptyset$ and a certain event $\bar{\emptyset}$. A map ()$^{P}$ : judg $\rightarrow$ events is an assignment $(t: A) \mapsto \mathbf{A}$ satisfying the following constraints:

$$
\text { if } \begin{aligned}
(M: C)^{P}= & \mathbf{C} \text { and }(x: D)^{P}=\mathbf{D} \text {, then }(\operatorname{mkc}(M, x): C \backslash D)^{P}=\mathbf{C} \cap \overline{\mathbf{D}} ; \\
& (\operatorname{postp}(x \mapsto M, N))^{P}=\emptyset .
\end{aligned}
$$

Let $d$ be a sequent calculus derivation of $x: H \triangleright t_{1}: C_{1}, \ldots, t_{n}: C_{n}$. We generate such a probabilistic assignment ()$^{P}:$ judg $\rightarrow$ events to the typed terms in $d$ as follows: $(i)$ in the case of an axiom we choose an event arbitrarily; (ii) in the conclusion of an inference, to the principal formula we assign an event as required by the constraints of Definition 2.1., given the assignments to premises, and we leave the assignment to the extra formulas unchanged.

Proposition 7. (Decomposition property) Let $d$ be a sequent calculus derivation of $x: H \triangleright t_{1}: C_{1}, \ldots, t_{n}: C_{n}$, let ()$^{P}:$ judg $\rightarrow$ events be an assignment to the typed terms in $d$ that satisfies the constraints of Definition 2.1. and suppose $\mathbf{H}, \mathbf{C}_{1}, \ldots, \mathbf{C}_{n}$ are assigned to $x: H \triangleright t_{1}: C_{1}, \ldots, t_{n}: C_{n}$. There are pairwise disjoint events $\mathbf{C}_{1}^{\prime} \subseteq \mathbf{C}_{1}, \ldots, \mathbf{C}_{n}^{\prime} \subseteq \mathbf{C}_{n}$ such that

$$
\left(\mathbf{C}_{1}^{\prime} \cup \ldots \cup \mathbf{C}_{n}^{\prime}\right) \cap \mathbf{H}=\mathbf{H} .
$$

The events $\mathbf{C}_{1}^{\prime} \cup \ldots, \mathbf{C}_{n}^{\prime}$ can be constructed from the dependencies of the terms $t_{1}, \ldots, t_{n}$.

Example 2. Consider the following very simple example:

$$
\frac{x: C \triangleright x: C \quad y: D \triangleright y: D}{x: C \triangleright \operatorname{mkc}(x, \mathrm{y}): C \backslash D, \mathrm{y}(x): D}
$$

If events $\mathbf{C}$ and $\mathbf{D}$ are assigned to the typed terms $x: C$ and $y: D$, respectively, we have the following inclusions:

$$
\frac{\mathbf{C} \subseteq \mathbf{C} \quad \mathbf{D} \subseteq \mathbf{D}}{\mathbf{C} \subseteq(\mathbf{C} \cap \overline{\mathbf{D}}) \cup \mathbf{D}}
$$

But we have equality only by assigning $\mathbf{C} \cap \mathbf{D}$ to y $(x): D$ as suggested by the dependency of y: $D$ on $x: C$. 
Proof of the decomposition property. By induction on $d$. See [9].

Remark 5. (i) Since events are assigned to expressions $t: X$ rather than to formulas $X$, if $t: X$ and $u: X$ occur in the same context then $(t: X)^{P}$ and $(u: X)^{P}$ are events that may or may not be disjoint of each other.

(ii) The common sense reading of the co-intuitionistic consequence relation $H \vdash C_{1}, \ldots, C_{n}$ is as follows.

If it is justified to make the hypothesis $H$, then it is justified to make the hypotheses $C_{1}, \ldots, C_{n}$.

The probabilistic interpretation gives a mathematical counterpart of this reading.

If the probability of the event $\mathbf{H}$ assigned to $x: H$ is greater than zero, then the conditional probability of the union of the events $\mathbf{C}_{1}, \ldots, \mathbf{C}_{n}$ assigned to $t_{1}: C_{1}, \ldots, t_{n}: C_{n}$, given $\mathbf{H}$ is equal to one.

The indexing of the terms $t_{1}, \ldots, t_{n}$ can be regarded as computational devices for verifying such an interpretation in the sense of the Decomposition Property.

\section{Conclusions}

We have examined the logical and philosophical standpoint of the logic for pragmatics initiated by Carlo Dalla Pozza and Claudio Garola with the paper [21]. It has the foundational ambition of showing the compatibility of intuitionism, as a logic of assertions and justifications, with classical logic, as a logic of propositions and truth, in their two-layers system, according to the principle change of logic, change of subject matter. We recognized that that system gives a primary role to classical logic and the use of classical logic in the meta-theory showed a reductive goal of reducing intuitionism to classical constructivism.

We did not examine the project of Justification Logic promoted by S. Artemov nor compared the massive work in that area with Dalla Pozza and Garola's contributions but recognized a similarity of intentions between the logic for pragmatics and some work in Justification Logic.

We asked whether a non-reductive representation of intuitionism in the logic for pragmatics was possible within that framework. We recognized the role of illocutionary forces to separate the extensional semantics of classical logic from the intensional aspects of illocutionary acts as represented in elementary sentences. This intensional quality gives room to relate the logic for pragmatics to modern aspects of intuitionism such as the Curry-Howard correspondence and the "propositions as types" approach. However, an essential point in Dalla Pozza and Garola's viewpoint is that the distinction between truth and provability cannot be blurred. Their commitment to classical semantics is clear: 
in elementary expressions classical proposition are intended within the scope of the operator of assertion or other illocutionary acts. A similar distinction between provability and an intuitionistic notion of truth should be retained if only intuitionistically acceptable propositions were allowed within the scope of illocutionary operators, ruling out "intrinsically undecidable" statements.

An application of the principles of the logic for pragmatics to a treatment of imperatives and norms, causal inferences and assertive reasoning was sketched as proposed in $[11,14]$. Such a logic, called $\mathbf{I L P}_{A O C}$, deals with the properties of impersonal acts of assertion and acts of obligation, and relates them through a notion of causal implication, regarded as a fragment of relevant logic. We only considered here the syntax of $\mathbf{I L P}_{A O C}$.

In Section 4.2 we reconsidered the treatment of bi-intuitionistic logic as developed in $[7,8,10]$ and gave a correct definition of the logic AHL that does represent the duality between intuitionism and co-intuitionism as a logic of assertions and objections. We ensure that the dual negations ()$^{\perp}$ that are internal to the language $\mathcal{L}^{A H}$ agree with the maps between the intuitionistic language $\mathcal{L}^{A}$ and the co-intuitionistic language $\mathcal{L}^{H}$. As a consequence, the conjecture that the categorical notion of chirality applies to the bi-intuitionistic logic AHL , as stated in [10], remains plausible.

Next we considered what appear as new illocutionary operators of expectation $\mathcal{E}$ and conjecture $\mathcal{C}$. They are defined through the $\mathbf{S} 4$ modal translation, by letting $\left(\mathcal{E} p^{*}\right)^{M}=\square \diamond p^{*}$ and $(\mathcal{C}(p))^{M}=\diamond \square p$. Then the Kripke model interpretation gives us the intuition of an expectation that in any situation we expect to find a situation where $p$ is true. Similarly the intuition of a conjecture is given by the possibility of a situation where $p$ is valid.

We show that the language built from elementary formulas $\mathcal{E} p^{*}$ with intuitionistic conjunction and implication but not intuitionistic disjunction is closed under the double negation rule. It is a classical result that a " $\square \diamond$ " translation of classical logic into $\mathbf{S} 4$ given by the map $p \mapsto \square \diamond p$ and the intuitionistic translation of the connectives except idisjunction, is sound and complete: we give a pragmatic interpretation of it. An entirely analogue discussion applies to the relations between Conjectures and Hypotheses.

A pragmatic treatment of co-intuitionism, seen as a logic of hypotheses, was then reviewed, considering work by Bellin et al. [7, 8, 9, 13, 10]. The difficulty of extending the BHK interpretation to co-intuitionism was also recognized, given the elusive nature of the notion of a "spark of evidence" which suffices to justify a hypothesis.

On the other hand, the term assignment to co-intuitionistic sequent calculus using a distributed calculus of coroutines "dual" to the simply typed $\lambda$ calculus, does provide a pragmatic intuition of how "parcels of evidence" may be treated in this logic. Essentially we have the notion of a computational context $\mathcal{S}_{x}$, consisting of the parallel composition of terms which are then assigned to a co-intuitionistic sequent $x: C \Rightarrow t_{1}: C_{1}, \ldots, t_{n}: C_{n}$. Such 
context are extended by merging two contexts in a cut, in a subtraction right and in adisjunction left inference, and are reduced in a subtraction left and a disjunction right inference. With subtraction left we associate an operator postpone $(z \mapsto \ell, u)$ that takes threads from $z: C$ to the list $\ell: D$ and sets them aside in a location $\bullet$, because such threads are incompatible with the information that $v: C \backslash D$.

$$
\frac{z: C \Rightarrow \ell: D, \mathcal{S}_{z}: \Upsilon}{v: C \backslash D \Rightarrow \operatorname{postpone}(z \mapsto \ell, v): \bullet, \mathcal{S}_{z(v)}: \Upsilon} \backslash \mathrm{L}
$$

These threads will be reused if in the normalization process the formula $C>$ $D$ is eliminated. As we can see, thus the calculus represents the dialectical process of extending and reducing the context of possible alternatives that are consequences of a single hypothesis. Moreover we have a computational notion of normalization that can simulate the simply typed $\lambda$ calculus with pairs and projections. We claim that this calculus gives a clear picture of what is done within the logic.

We give an informal presentation of the co-intuitionistic calculus of coroutines first. Then in the linear case we give a formal presentation.

It is evident that the distributed calculus of co-intuitionistic coroutines gives a static picture of a computations: it multiplies possible parallel avenue, some of which are first postponed then recovered. This is not surprising: the refutation of a hypothesis requires conclusive evidence against it, but deriving partial evidence for hypotheses from the evidence for one hypothesis cannot yield a conclusive evidence. The computational use of coroutines in the context of functional programming is gives a totally different view of the dynamics of computation. This indeed is the case with T. Crolard notion of coroutine, in the context of the $\lambda \mu$-calculus.

On the other hand a natural mathematical treatment of hypotheses is by using probability theory, as Carlo Dalla Pozza did in his work on Bayesian probability. We do have some information about how the probability of a hypothesis is related to the probabilities of its consequences. in a remark Indeed a probabilistic interpretation can be given to linear co-intuitionistic sequents decorated with a co-intuitionistic calculus of coroutines. Then one proves that the probability of a hypothesis $x: H$ is the same as the sum of the probabilities of the terms $t_{i}: H_{I}$ in the computational context $\mathcal{S}_{x}$, provided that the probability assignments are done correctly: this information can be given to us by the terms $t_{i}$ in the computational context $\mathcal{S}_{x}$. The result applies to the linear cointuitionistic calculus.

In conclusion, the logic for pragmatic has raised interesting problems in the consideration of the relations between intuitionistic logic, classical semantics and classical modal logic. Also it has given given interesting applications to representation of common sense reasoning and deontic logic. The problem of an interpretation of bi-intuitionistic logic which could represent the duality 
between intuitionistic and co-intuitionistic logic in proof-theoretic and categorical terms remains open, but the conjecture by Melliès that it can be represented as a dialogue chirality remains plausible.

\section{References}

1. N. Alechina, M. Mendler, V. de Paiva and E. Ritter. Categorical and Kripke Semantics for Constructive S4 Modal Logic, In Proceedings of the 15th International Workshop on Computer Science Logic, CSL01, L. Fribourg, Ed., Springer Lecture Notes in Computer Science, Vol. 2142, 2001, pp. 292-307.

2. I. Angelelli. The Techniques of Disputation in the History of Logic. The Journal of Philosophy 67:20, pp. 800-815, 1970.

3. S. Artemov. Explicit Provability and Constructive Semantics. The Bullettin of Symbolic Logic, 7. 1, 2001.

4. S. Artemov and R. Iemhoff. The basic Intuitionistic Logic of Proofs, The Journal of Symbolic Logic, 72, 2, 2007, pp.439-451.

5. F. Aschieri. Learning Based Realizability for HA + EM1 and 1-Backtracking Games: Soundness and Completeness, Annals of Pure and Applied Logic, 164, 6, June 2013, pp.591-617.

6. J. L. Austin. Philosophical Papers, Oxford University Press, 2nd edition, 1970.

7. G. Bellin and C. Biasi. Towards a logic for pragmatics. Assertions and conjectures. In: Journal of Logic and Computation, Volume 14, Number 4, 2004, pp. 473-506.

8. G. Bellin. Assertions, Hypotheses, Conjectures, Expectations: Rough-sets semantics and proof-theory. In: L. C. Pereira, E. H. Haeusler, V. de Paiva (eds) Advances in Natural Deduction. A Celebration of Dag Prawitzs Work, Trends in Logic 39, Springer Science+Business Media Dordrecht 2014, pp. 193-241.

9. G. Bellin. Categorical Proof Theory of Co-intuitionistic Linear Logic, Logical Methods in Computer Science Vol. 10(3:16) 2014, pp. 1-36 www.lmcs-online.org Sept. 232014.

10. G. Bellin, M. Carrara, D. Chiffi and A. Menti. Pragmatic and dialogic interpretations of bi-intuitionism. Parts 1 and 2. Logic and Logical Philosophy, http://dx.doi.org/10.12775/LLP.2014.011 and http://dx.doi.org/10.12775/LLP.2014.012, Published online June 23 2014.

11. G. Bellin and C. Dalla Pozza. A pragmatic interpretation of substructural logics. In Reflection on the Foundations of Mathematics (Stanford, CA, 1998), Essays in honor of Solomon Feferman, W. Sieg, R. Sommer and C. Talcott eds., Association for Symbolic Logic, Urbana, IL, Lecture Notes in Logic, Volume 15, 2002, pp. 139-163.

12. G. Bellin, M. Hyland, E.Robinson and C. Urban. Categorical Proof Theory of Classical Propositional Calculus Theoretical Computer Science Vol. 364, 2, November 2006, pp. 146-165.

13. G. Bellin and A. Menti. On the $\pi$-calculus and Co-intuitionistic Logic. Notes on Logic for Concurrency and $\lambda \mathrm{P}$ Systems, Fundamenta Informaticae 130, pp. 21-65, 2014. 
14. G. Bellin and K. Ranalter. A Kripke-style semantics for the intuitionistic logic of pragmatics ILP. In: Journal of Logic and Computation, Volume 13, Number 5, 2003, pp. 755-775.

15. C. Biasi and F. Aschieri. A Term Assignment for Polarized Bi-intuitionistic Logic and its Strong Normalization. In Fundamenta Informaticae, Special issue on Logic for Pragmatics, 84, 2, pp.185-205, 2008

16. G. M. Bierman. On Intuitionistic Linear Logic, PhD Thesis, University of Cambridge Computer Laboratory, Technical Report No. 346, 1994.

17. G. Brewka and T. Gordon. Carneades and Abstract Dialectical Frameworks: A Reconstruction. In: P. Baroni, M. Giacomin and G. Simari Computational Models of Argument, Proceedings of COMMA 2010, IOS Press, 2010.

18. T. Crolard. Subtractive logic, in Theoretical Computer Science 254,1-2, 2001, pp. 151-185.

19. T. Crolard. A Formulae-as-Types Interpretation of Subtractive Logic. In: Journal of Logic and Computation, vol.14(4), 2004, pp. 529-570

20. Curien, P.-L., Herbelin, H.: The duality of computation, Proceedings of the Fifth ACM SIGPLAN Inter- national Conference on Functional Programming (ICFP 00), Montreal, Canada, September 18-21, 2000, SIGPLAN Notices 35(9), ACM, 2000, ISBN 1-58113-202-6.

21. C. Dalla Pozza and C. Garola. A pragmatic interpretation of intuitionistic propositional logic, Erkenntnis 43. 1995, pp.81-109.

22. C.Dalla Pozza and A.Negro Il problema della demarcazione. Verificabilit, falsificabilit e confermabilit bayesiana a confronto Carocci, Roma, forthcoming.

23. M. Dummett. The Logical Basis of Metaphysics Cambridge, Mass.: Cambridge University Press, 1991.

24. M. Fairtlough and M. Mendler. Propositional Lax Logic, Information and Computation, 137, 1997, pp. 1-33.

25. J-Y. Girard. A new constructive logic: Classical logic, Mathematical Structures in Computer Science 1 (3) (1991) 255296.

26. V. Glivenko. Sur quelques points de la logique de M. Brouwer. In Bulletins de la classe des sciences, ser. 5, vol. 15:183188. Académie Royale de Belgique. 1929.

27. K. Gödel. Zur intuitionistischen Arithmetik und Zahlentheorie, Ergebnisse eines mathematischen Kolloquiums, 4: 3438, 1933. Reprinted in Gdel 1986, pp. 286295.

28. K. Gödel. Eine Interpretation des intuitionistischen Aussagenkalkls, Ergebnisse eines mathematischen Kolloquiums 4, 3940, 1933. Reprinted in Gdel 1986, pp. 300301 .

29. K. Gödel. Collected Works. I: Publications 19291936. S. Feferman, S. Kleene, G. Moore, R. Solovay, and J. van Heijenoort (eds.), Oxford: Oxford University Press.

30. T. F. Gordon and D. Walton. Proof burdens and standards. in: I. Rahwan and G. Simari eds., Argumentations in Artificial Intelligence, pp.239-258, 2009.

31. S. A. Kripke. Semantical analysis of modal logic I: Normal modal propostional calculi, Zeitschrift für Mathematische Logik und Grundlagen der Mathematik, 9, 1963.

32. F. W. Lawvere, Intrinsic co-Heyting boundaries and the Leibniz rule in certain toposes. In A. Carboni, M.C. Pedicchio and G. Rosolini (eds.), Category Theory (Como 1990), Lecture Notes in Mathematics 1488, Springer-Verlag 1991, pp. 279-297.

33. J.C.C. McKinsey and A. Tarski. Some theorems about the sentential calculi of Lewis and Heyting, Journal of Symbolic Logic 13, 1948, pp. 1-15, 
34. P-A. Melliès. A micrological study of negation. Manuscript, available at the author's web page.

35. P. Miglioli, U. Moscato, M. Ornaghi and U. Usberti, A constructivism based on Classical Truth. Notre Dame Jour. of Formal Logic, 30, 1989, 67-90.

36. D. Nelson. Constructible falsity, The Journal of Symbolic Logic, 14, pp. 16-26, 1949

37. M. Parigot. Lambda-Mu-Calculus: An Algorithmic Interpretation of Classical Natural Deduction. In A. Voronkov ed. Logic Programming and Automated Reasoning, Proceedings of the International Conference LPAR'92, St. Petersburg, Russia, July 15-20, 1992. Springer Lecture Notes in Computer Science 624, 1992, ISBN 3-540-55727-X, pp. 190-201.

38. M. Parigot. Strong Normalization for Second Order Classical Natural Deduction. In LICS 1993, Proceedings, Eighth Annual IEEE Symposium on Logic in Computer Science, 19-23 June 1993, Montreal, Canada, pp. 39-46.

39. J-H. Poincaré. Science et méthode, Paris Flammarion 1908.

40. D. Prawitz. Natural deduction. A proof-theoretic study. Almquist and Wikksell, Stockholm, 1965.

41. D. Pawitz. Ideas and results in proof theory. In Proceedings of the Second Scandinavian Logic Symposium, J. Fenstad ed., Studies in Logic and the Foundation of Mathematics, vol. 63, North Holland, 1971, pp. 235-307.

42. D. Prawitz. Meaning and Proofs: On the Conflict Between Classical and Intuitionistic Logic, Theoria 43 (1), 1977, pp. 2-40.

43. D. Prawitz. Meaning and Objectivity, in Meaning and Interpretation. Conference held in Stockholm, September 24-26 1998, D. Prawitz editor, Almqvist \& Wiksell, Sockholm 2002.

44. K. Ranalter. Reasoning about assertions, obligations and causality: on a categorical semantics for a logic for pragmatics, PhD Thesis, Queen Mary University of London and Universitá di Verona, 2008.

45. K. Ranalter. A semantic analysis of a logic for pragmatics with assertions, obligations, and causal implication, Fundamenta Informaticae 84(3-4), 2008. pages 443-470

46. C. Rauszer. Semi-Boolean algebras and their applications to intuitionistic logic with dual operations, in Fundamenta Mathematicae, 83, 1974, pp. 219-249.

47. C. Rauszer. Applications of Kripke Models to Heyting-Brouwer Logic, in Studia Logica 36, 1977, pp. 61-71.

48. G. Reyes and H. Zolfaghari, Bi-Heyting algebras, Toposes and Modalities, in Journal of Philosophical Logic, 25, 1996, pp. 25-43.

49. Y. Shramko. Dual intuitionistic logic and a variety of negations. The logic of scientific reseach, Studia Logica, 80 (2005): 347367. http://dx .doi.org/10.1007/s11225-005-8474-7

50. P. Selinger. Control categories and duality: on the categorical semantics of the lambda-mu calculus. Mathematical Structures in Computer Science, 11:207-260, 2001.

51. A. K. Simpson. The Proof Theory and Semantics of Intuitionistic Modal Logic, PhD Thesis, University of Edinbugh, 1994.

52. R. M. Smullyan and M. Fitting. Set Theory and the Continuum Problem, Claredon Press, Oxford 1996.

53. A. S. Troelstra and H. Schwichtenberg. Basic Proof Theory, Cambridge Tracts in Theoretical Computer Science 43, Cambridge University Press 1996. 


\section{APPENDIX I. Sequent Calculus AH-G1}

We give here the sequent calculus used in [10] for the logic $\mathbf{A H}$.

\subsection{Sequent calculus AH-G1.}

The sequent calculus AH-G1 has sequents of one of the forms

$$
\Theta ; \Rightarrow A ; \Upsilon \text { or } \Theta ; C \Rightarrow \Upsilon \Upsilon
$$

where the multiset $\Theta$ and $A$ are assertive formulas and the multiset $\Upsilon$ and $C$ are hypothetical formulas. We use the abbreviation

$$
\Theta ; \epsilon \Rightarrow \epsilon^{\prime} ; \Upsilon
$$

where exactly one of $\epsilon$ or $\epsilon^{\prime}$ is non-null. The inference rules of AH-G1 are in the following Tables.

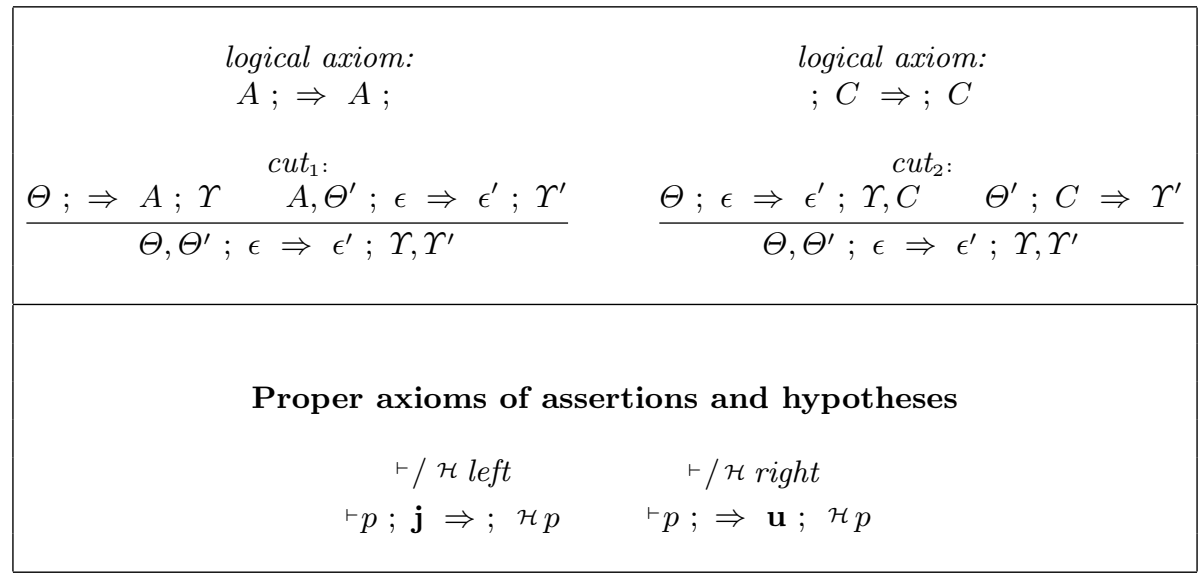

Table 2. Identity Rules 


\begin{tabular}{|cc|}
\hline contraction left & contraction right \\
$A, A, \Theta ; \epsilon \Rightarrow \epsilon^{\prime} ; \Upsilon$ & $\Theta ; \epsilon \Rightarrow \epsilon^{\prime} ; \Upsilon, C . C$ \\
\hline$A, \Theta ; \epsilon \Rightarrow \epsilon^{\prime} ; \Upsilon$ & $\Theta ; \epsilon \Rightarrow \epsilon^{\prime} ; \Upsilon, C$ \\
weakening left & weakening right \\
$\Theta ; \epsilon \Rightarrow \epsilon^{\prime} ; \Upsilon$ & $\Theta ; \epsilon \Rightarrow \epsilon^{\prime} ; \Upsilon$ \\
\hline$A, \Theta ; \epsilon \Rightarrow \epsilon^{\prime} ; \Upsilon$ & $\Theta ; \epsilon \Rightarrow \epsilon^{\prime} ; \Upsilon, C$ \\
\hline
\end{tabular}

Table 3. AH-G1 Structural Rules

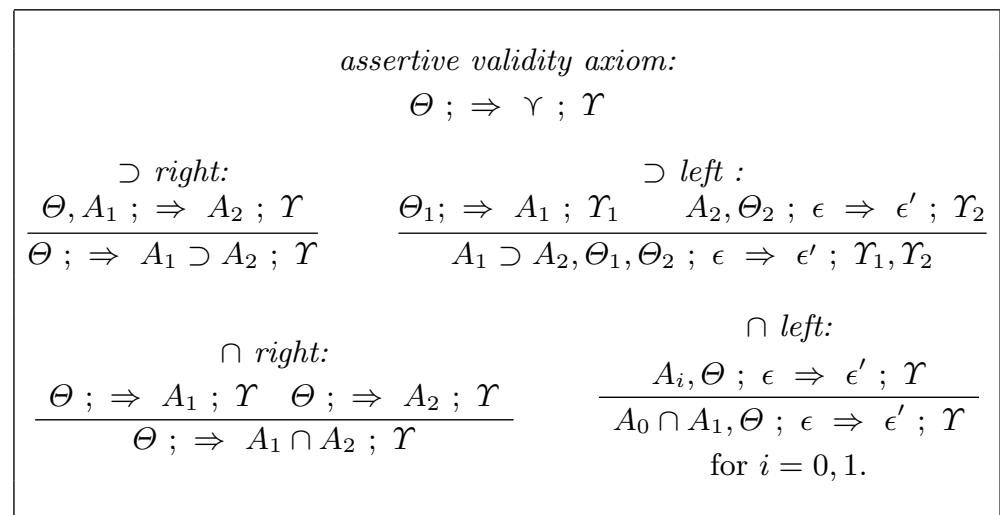

Table 4. AH-G1 intuitionistic rules

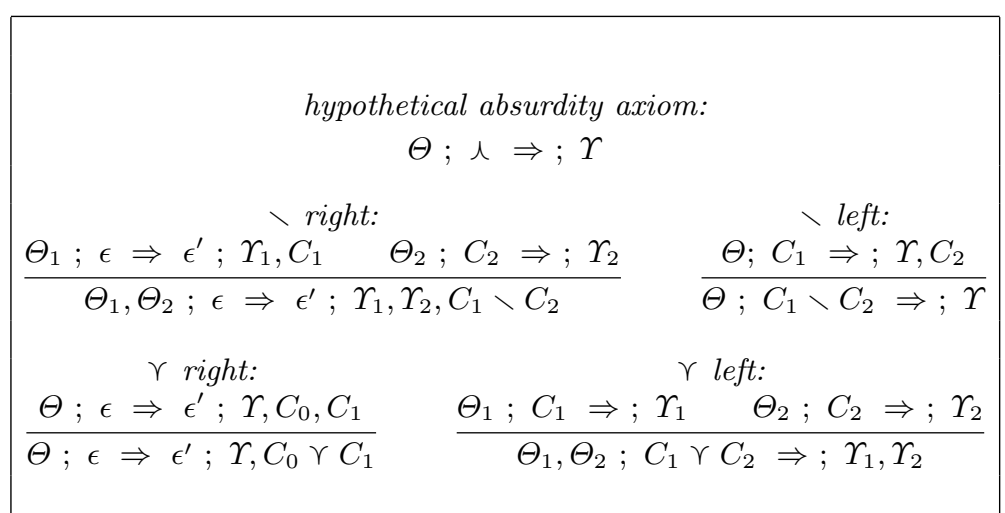

Table 5. AH-G1 Co-Intuitionistic Rules

Remark 6 . The syntax is chosen so that exactly one formula occurs in one of the focussed areas. If the right one is occupied by an assertive formula, we think of the sequent as part of an intuitionistic proof; similarly, when the left one is occupied by a hypothetical formula, the sequent is regarded as part of 


\begin{tabular}{|c|c|}
\hline $\begin{array}{c}h \perp_{a} \text { right: } \\
\Theta ; C \Rightarrow ; \Upsilon\end{array}$ & $\begin{array}{c}h \perp_{a} \text { left: } \\
\Theta ; \epsilon \Rightarrow \epsilon^{\prime} ; \Upsilon, C\end{array}$ \\
\hline$\Theta ; \Rightarrow C^{\perp} ; \Upsilon$ & $C^{\perp}, \Theta ; \epsilon \Rightarrow \epsilon^{\prime} ; \Upsilon$ \\
\hline $\begin{array}{c}{ }_{a} \perp_{h} \text { right: } \\
\Theta, A ; \epsilon \Rightarrow \epsilon^{\prime} ; \Upsilon\end{array}$ & $\begin{array}{c}{ }^{a} \perp_{h} \text { left: } \\
\Theta ; \Rightarrow A ; \Upsilon\end{array}$ \\
\hline$\Theta ; \epsilon \Rightarrow \epsilon^{\prime} ; \Upsilon, A^{\perp}$ & $\Theta ; A^{\perp} \Rightarrow ; \Upsilon$ \\
\hline $\mathbf{u} / \mathbf{j}$ left & $\mathbf{u} / \mathbf{j}$ right \\
\hline $\mathbf{u} ; \mathbf{j} \Rightarrow ;$ & $; \Rightarrow \mathbf{u} ; \mathbf{j}$ \\
\hline
\end{tabular}

Table 6. AH-G1 Duality Rules

a co-intuitionistic proof. The duality rules operating on focussed areas switch between the two interpretations.

\subsection{Dialogue chirality.}

The concept of chirality, due to Paul-André Melliès [34], provides an abstract characterisation of the interactions within bi-intuitionism and the emergency of classical behaviour in them. We have a pair of dual monoidal categories $(\mathcal{A}, \mathcal{B})$, where the "intuitionistic" structure $\mathcal{A}$ is cartesian closed, and $\mathcal{B}$ is "cointuitionistic".

Definition 12. A dialogue chirality on the left is a pair of monoidal categories $(\mathcal{A}, \wedge$, true $)$ and $(\mathcal{B}, \vee$, false $)$ equipped with an adjunction

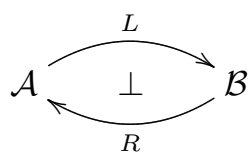

whose unit and counit are denoted as

$$
\eta: I d \quad \rightarrow \quad R \circ L \quad \epsilon: L \circ R \rightarrow I d
$$

together with a monoidal functor

$$
(-)^{*}: \mathcal{A} \rightarrow \mathcal{B}^{o p}
$$

and a family of bijections

$$
\chi_{m, a, b}:\langle m \wedge a \mid b\rangle \rightarrow\left\langle a \mid m^{*} \vee b\right\rangle
$$

natural in $m, a, b$ (curryfication). Here the bracket $\langle a \mid b\rangle$ denotes the set of morphisms from a to $R(b)$ in the category $\mathcal{A}$ : 


$$
\langle a \mid b\rangle=\mathcal{A}(a, R(b)) .
$$

The family $\chi$ is moreover required to make the diagram

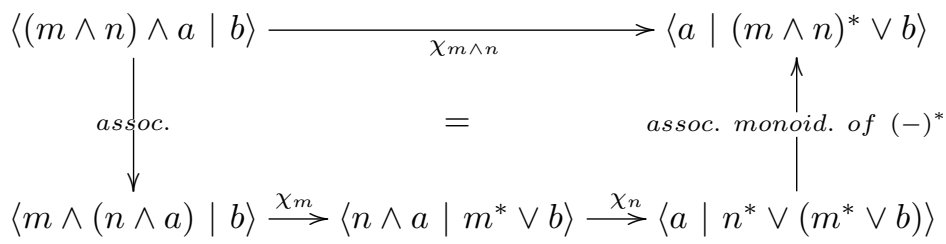

commute for all objects $a, m, n$, and all morphisms $f: m \rightarrow n$ of the category $\mathcal{A}$ and all objects $b$ of the category $\mathcal{B}$.

Thus in a chirality we have a sort of "De Morgan definition" of implication.

Consider the "sketch of proof" given in [10]. Paul-André Melliès' notion of dialogue chirality [34] requires two monoidal categories $(\mathcal{A}, \wedge$, true $)$ and $(\mathcal{B}, \vee$, false $)$, a monoidal contravariant functor ()$^{*}: \mathcal{A} \rightarrow \mathcal{B}^{o p}$ and an adjunction $L \dashv R$ between functors $L: \mathcal{A} \rightarrow \mathcal{B}$ and $R: \mathcal{B} \rightarrow \mathcal{A}$. We claimed that polarized bi-intuitionism AHL with the sequent calculus AH-G1 constitutes a dialogue chirality.

We suggested to define $\mathcal{A}$ as the free category on the language $\mathcal{L}^{A}$ of Int with equivalence classes of $\mathbf{A H}-\mathbf{G} \mathbf{1}$ intuitionistic sequent derivations as morphisms and $\mathcal{B}$ as the free category on the language $\mathcal{L}^{H}$ of co-Int with co-intuitionistic sequent derivations as morphisms. We gave $\mathbf{A H - G 1}$ proofs that can be interpreted as the unit and the co-unit of an adjunction between suitable functors $L=\diamond$ and $R=\square$ namely, the proof of Proposition 4 (ii) in Section 4.3. Then we claimed that the operation ()$^{\perp}: \mathcal{A} \rightarrow \mathcal{H}^{o p}$ is functorial. In a logic of assertions and hypotheses by the Remark 1 with the definition (1) in Section 1 adopted in [10], the metatheoretic map ( $)^{\perp}$ is not defined on elementary formulas of $\mathcal{L}^{A}$. Hence the conjecture is false. But in a logic of assertions and objections with the official definition 3 in Section 4.2 the metatheoretic map ()$^{\perp}$ is total, hence there is no obvious error in our suggested approach to Melliès conjecture. 